\title{
Encoding of Head Direction by Hippocampal Place Cells in Bats
}

\author{
Alon Rubin, Michael M. Yartsev, and Nachum Ulanovsky \\ Department of Neurobiology, Weizmann Institute of Science, Rehovot 76100, Israel
}

Most theories of navigation rely on the concept of a mental map and compass. Hippocampal place cells are neurons thought to be important for representing the mental map; these neurons become active when the animal traverses a specific location in the environment (the "place field"). Head-direction cells are found outside the hippocampus, and encode the animal's head orientation, thus implementing a neural compass. The prevailing view is that the activity of head-direction cells is not tuned to a single place, while place cells do not encode head direction. However, little work has been done to investigate in detail the possible head-directional tuning of hippocampal place cells across species. Here we addressed this by recording the activity of single neurons in the hippocampus of two evolutionarily distant bat species, Egyptian fruit bat and big brown bat, which crawled randomly in three different open-field arenas. We found that a large fraction of hippocampal neurons, in both bat species, showed conjunctive sensitivity to the animal's spatial position (place field) and to its head direction. We introduced analytical methods to demonstrate that the head-direction tuning was significant even after controlling for the behavioral coupling between position and head direction. Surprisingly, some hippocampal neurons preserved their head direction tuning even outside the neuron's place field, suggesting that "spontaneous" extra-field spikes are not noise, but in fact carry head-direction information. Overall, these findings suggest that bat hippocampal neurons can convey both map information and compass information.

\section{Introduction}

The hippocampus is crucial for spatial memory and navigation (Andersen et al., 2007). Hippocampal "place cells" are neurons activated when an animal enters a restricted region of the environment, the "place field" (O'Keefe and Nadel, 1978; Wilson and McNaughton, 1993; Moser et al., 2008). The positional signal carried by place cells is not sufficient, however, for implementing a full navigational system (Moser et al., 2008). Other essential components of navigation must include a measurement of distance, and a compass-constituting together the key ingredients of the "map and compass" model of navigation (Kramer, 1953). The neural measurement of distance is thought to be implemented by "grid cells" (Hafting et al., 2005; Barry et al., 2007). The neural compass is implemented by "head-direction cells" (Taube et al., 1990; Wiener and Taube, 2005), neurons that encode the directionality of the animal's head, and are found in multiple brain regions surrounding the hippocampus (Taube, 2007; Boccara et al., 2010)—including in the entorhinal cortex, where a head-direction signal is sometimes represented conjunc-

\footnotetext{
Received Nov. 21, 2012; revised Nov. 19, 2013; accepted Nov. 26, 2013.

Author contributions: A.R. and N.U. designed research; A.R., M.M.Y., and N.U. performed research; A.R. contributed unpublished reagents/analytic tools; A.R. analyzed data; A.R. and N.U. wrote the paper.

This study was supported by research grants from the European Research Council (ERC-NEUROBAT), Israel Science Foundation (ISF 1017/08 and ISF 1319/13), and Minerva Foundation to N.U., and by a Lev-Tzion predoctoral excellence fellowship to M.M.Y. We thank A. Treves, D. Derdikman, and L. Las for comments on this manuscript; B. Pasmantirer and G. Ankaoua for mechanical designs; M. Weinberg for veterinary oversight; R. Eilam and C. Ra'anan for histology; and C. Moss for sharing big brown bat data that were recorded in her laboratory by N.U.

Correspondence should be addressed to Nachum Ulanovsky, Department of Neurobiology, Weizmann Institute of Science, Rehovot 76100, Israel. E-mail: nachum.ulanovsky@weizmann.ac.il.

DOI:10.1523/JNEUROSCI.5393-12.2014

Copyright $\odot 2014$ the authors $\quad 0270-6474 / 14 / 341067-14 \$ 15.00 / 0$
}

tively with the distance signal, in "grid $\times$ head-direction cells" (Sargolini et al., 2006). However, the picture in the hippocampus proper seems to be quite different.

Despite early reports that hippocampal place cells may exhibit head-direction tuning (Fig. 1A; Eichenbaum et al., 1989; Wiener et al., 1989), later studies suggested that this kind of directional modulation can be explained as a byproduct of the behavioral coupling between location and head direction (Fig. 1B). For example, whenever the animal's head is located close to a corner of the arena, the animal cannot be facing away from the corner, because this requires its body to be beyond the wall, which is physically impossible. Therefore, a hippocampal neuron having a place field in that corner would display an apparent head directionality, because when the animal's head is inside the place field, and spikes are being emitted, the animal would also necessarily face the direction of the corner (Muller et al., 1994; Burgess et al., 2005). Because of this behavioral-coupling problem, the prevailing view is that place cells in the hippocampus do not have true head-direction tuning.

We set out to examine this question in a novel animal model: the bat. Bats are mammals in which all the main neural components of a navigational system-place cells, grid cells, and head direction cells-have been recently described (Ulanovsky and Moss, 2007, 2011; Yartsev et al., 2011). We developed here new analytical methods to examine the possible head-direction tuning of hippocampal place cells. These new analyses were applied to data that we collected from two different bat species, performing a random-foraging task in three types of open-field arenas (Ulanovsky and Moss, 2007, 2011; Yartsev et al., 2011). We found that a large fraction of hippocampal place cells exhibited signifi- 


\section{A Rat - Wiener et al. (1989)}

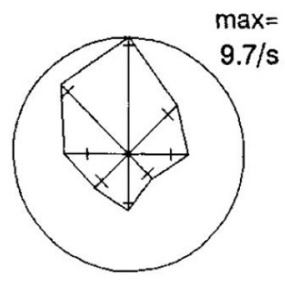

B Rat - Muller et al. (1994)

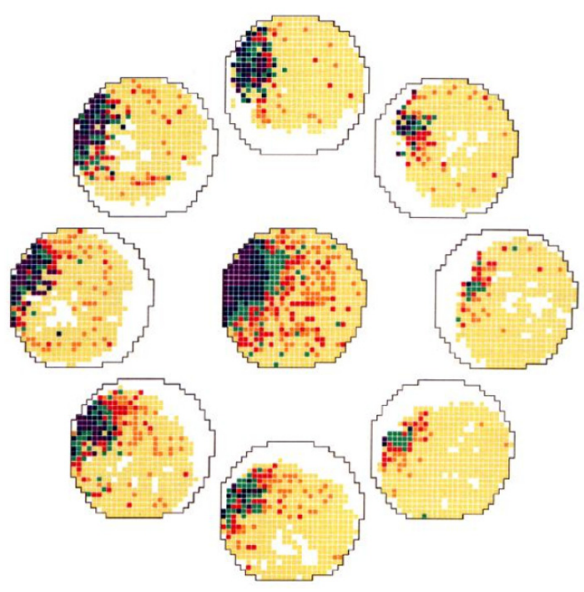

Figure 1. Head-direction tuning of hippocampal place cells in rodents, as reported in previous studies of rat CA1 neurons. $A$, Example of a rat place cell tuned to the animal's head direction (polar plot of the firing rate in each of the 8 cardinal directions). Adapted with permission from Wiener et al. (1989). B, Example demonstrating that the head-direction tuning of a rat place cell may be a byproduct of the animal's location. Shown in the center is the firing-rate map, surrounded by eight maps calculated separately for eight different head-direction bins. Note the area of the place field is not evenly covered when the animal is facing at different directions (white pixels, noncovered area), resulting in a head-direction tuning that may possibly be artifactual. Adapted with permission from Muller et al. (1994). This type of finding has led to the currently widely accepted notion that place cells recorded in $2 \mathrm{D}$ environments are not truly tuned to the animal's head direction.

cant head-direction tuning. Interestingly, head-direction selectivity was exhibited not only by spikes emitted within the place field, but also by "spontaneous spikes" emitted outside the place field; these spikes, which are commonly treated as "noise spikes," carried a surprisingly strong head-direction signal. These results suggest that hippocampal place cells carry both map and compass information.

\section{Materials and Methods}

Subjects and behavioral training. Neural activity was recorded from five male bats: three adult Egyptian fruit bats, Rousettus aegyptiacus (weight 156-171 g at implantation), and two adult big brown bats, Eptesicus fuscus (weight 15-18 g at implantation). Experimental procedures were approved by the Institutional Animal Care and Use Committees of the Weizmann Institute of Science (Egyptian fruit bats) and the University of Maryland (big brown bats), and are detailed elsewhere (Ulanovsky and Moss, 2007; Yartsev et al., 2011). In brief, five bats were trained to randomly crawl inside one of three experimental arenas; all arenas consisted of a $2 \mathrm{D}$, open-field rectangular surface, either tilted or placed horizontally: (1) a small, $68 \times 73 \mathrm{~cm}$ arena with $40 \mathrm{~cm}$ high walls, tilted $70^{\circ}$ above the horizontal plane (big brown bats, $n=2$ bats); (2) a small, $62 \times 62 \mathrm{~cm}$ horizontal arena with $50 \mathrm{~cm}$ high walls (Egyptian fruit bats, $n=1$ ); and (3) a large, $117 \times 117 \mathrm{~cm}$ horizontal arena with $70 \mathrm{~cm}$ high walls (Egyptian fruit bats, $n=2$ ). In all cases, the camera was positioned perpendicular to the arena surface (the camera was vertically overhead in setups 2 and 3 , and was pointing $20^{\circ}$ below the horizon in setup 1). Bats were trained to crawl in search of randomly scattered food (pieces of banana for the Egyptian fruit bats, and mealworms for the insect-eating big brown bats). In all three experimental setups, the walls of the arena were marked asymmetrically with visual landmarks, as described previously (Ulanovsky and Moss, 2007; Yartsev et al., 2011). Behavioral recording sessions lasted 15-30 min and were held under dim light conditions of 1-2 lux (Ulanovsky and Moss, 2007; Yartsev et al., 2011). At the start and end of each recording day the arena was cleaned with alcohol to remove odors.

Surgery and recording techniques. All surgical and recording procedures were as described previously (Ulanovsky and Moss, 2007; Yartsev et al., 2011). Briefly, after completion of training, bats were implanted with a four-tetrode microdrive (weight $2.1 \mathrm{~g}$; Neuralynx), loaded with tetrodes constructed from four strands of insulated wire (17.8 $\mu \mathrm{m}$ diameter platinum-iridium wire for recordings in Egyptian fruit bats; $12.7 \mu \mathrm{m}$ nichrome wire for big brown bats). Tetrodes were gold plated to reduce wire impedance to $0.3-1.0 \mathrm{M} \Omega$ (at $1 \mathrm{kHz}$ ). The microdrive was implanted above the right dorsal hippocampus, and tetrodes were slowly lowered toward the CA1 pyramidal layer; positioning of tetrodes in the layer was provisionally determined by the presence of high-frequency field oscillations ("ripples") and associated neuronal firing, and was later verified histologically (Ulanovsky and Moss, 2007; Yartsev et al., 2011). For each bat, one tetrode was left in an electrically quiet zone and served as a reference, and the remaining three tetrodes served as recording probes. During recordings, a unity-gain preamplifier (HS-16 or HS-18; Neuralynx) was attached to a connector on the microdrive. Signals were amplified $(\times 1400-5000)$ and bandpass filtered $(600-6000 \mathrm{~Hz}$, Lynx-8 or Digital Lynx; Neuralynx), and a voltage threshold was used for collecting $1 \mathrm{~ms}$ spike waveforms, sampled at $32 \mathrm{kHz}$. A video tracker (Neuralynx) recorded the positions of two light-emitting diodes (LEDs) on the bat's head; these two diodes had different colors and were oriented perpendicular to the long axis of the head. The center of mass of the diodes was used to estimate the bat's $x-y$ position, and the perpendicular vector was used to estimate the animal's head direction (direction of the nose). Behavioral sessions were flanked with sleep sessions; data were collected continuously throughout all the sessions in each recording day.

Spike sorting. All spike-sorting procedures are identical to those described previously (Ulanovsky and Moss, 2007; Yartsev et al., 2011). Briefly, spike waveforms were sorted on the basis of their relative energies and amplitudes on different channels of each tetrode (SpikeSort3D; Neuralynx). Well isolated clusters of spikes were manually encircled ("cluster-cutting"), and a refractory period $(<2 \mathrm{~ms})$ in the interspike interval histogram was verified. Putative pyramidal cells were identified based on the following: (1) narrow spike waveform followed by long hyperpolarization, (2) mean firing rate $<5 \mathrm{~Hz}$, (3) interspike interval histograms indicating complex-spike bursts, and (4) the simultaneous recording of other complex-spike cells (Ulanovsky and Moss, 2007; Yartsev et al., 2011). We considered here only "behaviorally active neurons," that is, neurons that emitted $\geq 50$ spikes during the behavioral session.

Firing-rate maps, spatial and directional information, and Rayleigh vector. To analyze firing-rate maps of hippocampal neurons, we divided the recording arena into $16 \times 16$ square bins (same number of bins for all three arenas), and computed two maps: (1) the time spent in each spatial bin, and (2) the spike count per bin. These two maps (time-spent and spike-count) were then individually smoothed using a Gaussian kernel with standard deviation of $\sigma=1.5$ bins (the kernel was truncated after two bins on each side, i.e., kernel size was $5 \times 5$ bins). The firing-rate map was then computed for each neuron by dividing bin by bin the two smoothed maps of spike count and time spent. Unvisited bins (where total time spent, before smoothing, was $<300 \mathrm{~ms}$ ) were discarded from analysis and are shown as white bins on plots of firing-rate maps.

The peak firing rate was defined as the highest observed firing rate in any of the bins of the firing-rate map. Spatial coherence for each neuron was estimated as the $Z$-transformed correlation between the original (unsmoothed) firing-rate map and the firing rates averaged across the eight neighbors of each bin (Muller et al., 1987; Ulanovsky and Moss, 2007). The spatial information, in bits per spike, was computed for the 
unsmoothed firing-rate map of each cell, as described previously (Skaggs et al., 1993; Ulanovsky and Moss, 2007; Yartsev et al., 2011):

$$
\text { Spatial information (bits/spike) }=\sum p_{i}\left(r_{i} / \bar{r}\right) \log _{2}\left(r_{i} / \bar{r}\right)
$$

where $r_{i}$ is the firing rate of the cell in the $i$ th bin of the place field, $p_{i}$ is the probability of the animal being in the $i$ th bin (time spent in $i$ th bin/total session time), $\bar{r}$ is the overall mean firing rate, and $i$ is running over all the bins where the bat spent $\geq 300 \mathrm{~ms}$. Place cells were defined based on a shuffling procedure (Langston et al., 2010; Wills et al., 2010; Yartsev et al., 2011). Specifically, for each recorded CA1 neuron, the entire sequence of spikes was time shifted by a random (uniformly distributed) interval between $10 \mathrm{~s}$ and the duration of the recording session minus $10 \mathrm{~s}$; the end of the session was wrapped to the beginning. This preserved the spike number and the temporal structure of the neuron's firing pattern, but dissociated the time of spiking from the animal's actual trajectory. This procedure was repeated 1000 times for each neuron, and the spatial information was computed for each repetition. A neuron was defined as a place cell if its information per spike exceeded the 95th percentile of the shuffled distribution for this neuron. Of the $n=108$ behaviorally active hippocampal CA1 pyramidal neurons, reported in the current study, $n=86$ cells $(80 \%)$ were place cells, according to the shuffling criterion.

The head-direction tuning of a neuron was computed as follows. We binned the directional data in 10 bins of $36^{\circ}$ each, and computed the firing rate in each bin by dividing the number of spikes in that bin by the time the animal spent in that bin. No smoothing was applied to the resulting circular distribution (because the usage of such large $36^{\circ}$ bins effectively already implements quite substantial smoothing). The peak firing rate was defined as the highest rate in the head-direction tuning curve. The directionality of the tuning curve was quantified by computing the Rayleigh vector length of the circular distribution, using the following definition (Zar, 2010):

$$
\frac{\pi}{n \cdot \sin \left(\frac{\pi}{n}\right)} \sum_{j=1}^{n} r_{\theta_{j}} e^{-i \theta_{j}} / \sum_{j=1}^{n} r_{\theta_{j}},
$$

where $n$ is the number of circular head-direction bins, $\theta_{j}$ is the direction in radians of the $j$ th circular bin (namely, $2 \pi j / n$ ), and $r_{\theta_{j}}$ is the average firing rate for a given head direction.

We applied a shuffle-based significance test for the Rayleigh vector length, using the same approach as described above for the spatial information. In addition to the cell-specific shuffles (the individual shuffles for each neuron), we also compared the Rayleigh vector length of each neuron to the pooled shuffles of all the neurons in the recorded population (Langston et al., 2010; Wills et al., 2010). This "population-shuffle" gave rather similar results to the cell-specific shuffles; subsequently, individual cell-specific shuffles were used throughout the paper.

In-field and out-of-field tuning for head direction. To determine whether hippocampal place cells maintained their head-direction tuning outside the neuron's main firing field, we defined for each place-cell two regions of interest: "in-field," the main region of activity inside the arena, and "out-of-field," the rest of the arena. Specifically, we first computed the convex hull encompassing the set of contiguous spatial bins in which the firing rate was larger than $50 \%$ of the peak firing rate (Fig. $6 \mathrm{~A}$, left, thin gray line). Then, to make sure we include also the "tails" of the place field, we expanded the convex hull by two spatial bins in each direction, surrounding the place field, and have defined the in-field as this expanded area (Fig. 6A, left, thick gray line). The out-field region was defined as the area outside of this thick gray line. Only place cells with a single place field were included in this analysis (Fig. 6A-D); furthermore, we included in this analysis only place cells for which all the headdirection bins contained data for both the in-field and out- field tuning curves ( $n=60$ cells). Additionally, for the analysis in Figure 6, $E$ and $F$, we compared the preferred head directions for pairs of place fields that belonged to the same cell (this analysis included nonsignificant place cells).
Place-field radius (Fig. $8 H, x$-axis) was estimated as $\vee S / \pi$, where $S$ is the area of the in-field region ( $\sqrt{ } S / \pi$ is formally equal to the place-field radius if the field is circular).

The Rayleigh vector length for the out-field region was computed only for place cells with a single field that, additionally, emitted $>50$ spikes in the out-field region ( $n=54$ cells).

Location-specific head-direction analysis. To further examine whether the head-direction tuning for each cell was maintained throughout the available environment, we developed the following method (Figs. 7, 8; see also main text): The behavioral arena was divided into $8 \times 8$ spatial bins (Fig. $8 A$ ), and we calculated the head-direction tuning curves for each spatial bin, and then extracted the corresponding Rayleigh vector lengths and preferred head directions, using $45^{\circ}$ directional bins. Only spatial bins for which at least seven of the eight head-direction bins contained each a minimal time spent of $300 \mathrm{~ms}$ were included in this analysis. In those cases in which only seven bins included a sufficient amount of data, the firing rate of the eighth directional bin was taken as the mean firing rate of its two neighboring bins. Spatial locations (spatial bins), which contained fewer than seven valid head-direction bins, were excluded from this analysis (Fig. 8C,D; white bins). To quantify the consistency of directional tuning across space, we computed a "consistency index," by calculating the "location-specific Rayleigh vector length" for the histogram of the preferred head directions over the valid spatial bins (i.e., computing the Rayleigh vector length for the histograms in Fig. 8E). For each neuron, the consistency index was thus defined as follows:

$$
\frac{\pi}{n \cdot \sin \left(\frac{\pi}{n}\right)} \sum_{j=1}^{n} n_{\theta_{j}} e^{-i \theta_{j}} / \sum_{j=1}^{n} n_{\theta_{j}},
$$

where $n$ is the number of head-direction sectors, and $n_{\theta_{j}}$ is the number of spatial bins whose preferred head direction was $\theta_{j}$.

Reconstruction of head-direction tuning curves and firing-rate maps. To further elucidate the relations between the spatial firing-rate maps and head-direction tuning curves, we asked to what extent the firing-rate map can be used to reconstruct the head-direction curve, or vice versa. This was done to test whether the head-direction tuning curve could be a byproduct of the location of the firing field within the arena, as suggested previously (Muller et al., 1994), but also conversely, whether the firingrate map could have possibly emerged from the head-direction properties of the neuron.

The reconstructed head-direction firing rate $\hat{r}(\theta)$ that we would expect to obtain purely from the firing-rate map was calculated as follows:

$$
\hat{r}(\theta)=\sum_{x, y}(p(x, y, \theta) \cdot r(x, y)) / \sum_{x, y} p(x, y, \theta),
$$

where $p(x, y, \theta)$ is the fraction of time that the animal has spent at location $(x, y)$ with its head pointing toward direction $\theta$, and $r(x, y)$ is the mean firing rate for location $(x, y)$. Conversely, the reconstructed spatial firing rate $\hat{r}(x, y)$ that we would expect to get purely from the head-direction tuning curve was calculated as follows:

$$
\hat{r}(x, y)=\sum_{\theta}(p(x, y, \theta) \cdot r(\theta)) / \sum_{\theta} p(x, y, \theta),
$$

where $p(x, y, \theta)$ is the fraction of time that the animal has spent in location $(x, y)$ with its head pointing toward direction $\theta$, and $r(\theta)$ is the mean firing rate for head-direction $\theta$. This analysis allowed us to examine the relation between the observed and reconstructed firing-rate maps and head-direction tuning curves (Fig. 9).

Maximum-likelihood method for estimating the head direction. We compared our analysis to a previously published maximum-likelihood method for estimating place- and head-direction tuning (Burgess et al., 2005; we used the original MATLAB code published by these authors). To this end, we first analyzed simulated data using both the maximumlikelihood and our location-specific head-direction estimate; these were compared in Figure 7. The maximum-likelihood method estimates the most probable place-field and head-direction tuning given the firing pattern of the neuron throughout the recorded behavioral session, assuming Poisson firing and a multiplicative separability of place tuning 
and head-direction tuning. In the simulations, we created cells with nonuniform coverage over the arena. Arrows in Figure $7 B$, in each of the four marked spatial zones (at the four corners), denote the animal has spent more time facing the direction indicated by the arrow (fourfold behavioral bias as compared with the opposite direction); in the remainder of the arena ("+" zone) the time spent at each direction was distributed uniformly. In all the simulated cells, the firing rate in-field was $3 \mathrm{~Hz}$ and out-field $0.3 \mathrm{~Hz} ; 30 \mathrm{~min}$ of Poisson firing with these rates were simulated, which is equivalent to the duration of a typical behavioral session in the actual neural data. Note that the maximum-likelihood method reconstructed well the head direction in the two spatially uniform cases (Fig. 7, cells 1 and 3, compare $C, B$ ), but failed in the spatially nonuniform case (cell 2, compare $C, B$ ); in contrast, the per-spatial-pixel method reconstructed well the head-direction distributions in all cases, albeit with some noisiness (compare $D, B$; see more in the main text).

As a further validation of our per-pixel method, we simulated a neuron with no head-direction tuning, but under conditions of a behavioral bias, whereby a specific head direction was looked at (behaviorally) four times more often than the opposite direction. Running 10,000 realizations of this simulation showed that the preferred head direction was indeed distributed quite uniformly (coefficient of variation; $\mathrm{CV}=0.06$ ), as expected. The estimated Rayleigh vector lengths were very low (median Rayleigh vector length for $3 \mathrm{~Hz}$ firing rate $=0.15$, with $95 \%$ of the pixels showing Rayleigh vector length $<0.3$ ), as expected.

Histology. Histology was done as described previously (Ulanovsky and Moss, 2007; Yartsev et al., 2011). In brief, tetrodes were not moved after the final recording session. The bat was given an overdose of sodium pentobarbital and, with tetrodes left in situ, was perfused transcardially using a flush of $50 \mathrm{ml}$ PBS followed by $200 \mathrm{ml}$ of fixative (4\% paraformaldehyde $+0.1 \mathrm{M}$ PBS). The brains were removed and stored in fixative. Subsequently, a coronal block was embedded in paraffin, and then $10 \mu \mathrm{m}$ coronal sections were cut. For the Egyptian fruit bats every third section was mounted on glass slides and for the big brown bats every second section was mounted, resulting in $30 \mu \mathrm{m}$ or $20 \mu \mathrm{m}$ intervals between adjacent mounted sections, respectively. The sections were then Nissl stained with cresyl violet, and coverslipped. A light microscope fitted with a digital camera was used to determine tetrode placement in the CA1 pyramidal layer of dorsal hippocampus.

\section{Results}

Hippocampal place cells of two bat species encode head-direction information in open-field environments

Hippocampal place cells are neurons that become active when the animal traverses a certain location in the environment called the place field (O'Keefe and Dostrovsky, 1971; O'Keefe, 1976; O'Keefe and Nadel, 1978). We asked whether, in addition to this positional "map" information, place cells might carry also headdirectional, or "compass" information. To address this question, we conducted experiments in two bat species (Egyptian fruit bats and big brown bats) in three different behavioral setups, in all of which the animals where trained to randomly crawl on an openfield, $2 \mathrm{D}$ surface, in search of food reward. The head's location and azimuthal direction were measured using two colored LEDs, and the activity of individual hippocampal CA1 neurons was recorded using standard tetrode-based techniques (see Materials and Methods). A total of $n=108$ well isolated, active pyramidal neurons were recorded from five bats for this study: $n=42$ cells from two Egyptian fruit bats in a large arena (Yartsev et al., 2011), $n=16$ cells from one Egyptian fruit bat in a small arena (new recordings), and $n=50$ neurons from two big brown bats in a small arena (Ulanovsky and Moss, 2007; Materials and Methods).

We first characterized the head-direction tuning of hippocampal place cells using standard analysis methods, namely, by measuring the distribution of head directions when spikes occurred, and normalizing it by the total distribution of visitation times at each head direction (Taube et al., 1990; Sargolini et al.,
2006; Hargreaves et al., 2007; see Materials and Methods). Examples of the spatial firing-rate maps of hippocampal place cells, and their corresponding head-direction tuning curves, are shown in Figure 2A (Egyptian fruit bat: large arena), Figure 2B (Egyptian fruit bat: small arena), and Figure $2 C$ (big brown bat: small arena). Most of these example neurons had clear spatial place fields, but they also exhibited clear head-direction tuning curves (Fig. $2 A-C$, gray curves).

To quantify the directionality of the head-direction tuning curve of each cell, we used the Rayleigh vector length, which is a standard index used to characterize the tuning of head-direction cells in rats (Boccara et al., 2010; Langston et al., 2010; Wills et al., 2010) and bats (Yartsev et al., 2011). The Rayleigh vector length value is indicated near each of the example cells in Figure 2, $A-C$ (numbers in green on the right), demonstrating that many hippocampal CA1 cells had Rayleigh vector lengths of $0.3-0.4$, or higher, which are values considered to indicate tuning to head direction (Langston et al., 2010; Wills et al., 2010; Yartsev et al., 2011). A Monte Carlo shuffling procedure (see Materials and Methods) was used to assess the significance of head-direction tuning for hippocampal neurons (Fig. 2D). This procedure was performed for the entire population (Fig. $2 D$, red line; giving a global significance threshold of 0.34 when comparing each neuron to shuffling of the entire population), as well as for each individual neuron (gray bars; significantly tuned neurons at the $p=0.05$ significance level). More than half of the active hippocampal cells were significantly tuned to head direction (Fig. $2 D$, gray bars; $56 / 108$, or $52 \%$ of the neurons were directionally tuned). This fraction of directionally tuned neurons was approximately similar between the two species and the three experimental setups (27/50 of the cells were significantly tuned in big brown bat, small arena; 18/42 in Egyptian fruit bat, large arena; and 11/16 in Egyptian fruit bat, small arena). Among the population of place cells $(n=86 / 108$ cells with significant spatial information, see Materials and Methods), the proportion of neurons with significant head-direction tuning was even higher: $50 / 86$, or $58 \%$ of the place cells were significantly tuned to head direction.

Further, when plotting for all the neurons their Rayleigh vector length versus their spatial information (an index of spatial tuning), the population of hippocampal neurons did not cluster into distinct subpopulations of place cells versus head-direction cells, but rather exhibited a continuum (Fig. 2E). This continuous scatter was rather similar for the two bat species-Egyptian fruit bats and big brown bats (Fig. 2E, black dots and red dots)-indicating that hippocampal neurons in both of these bat species exhibited similar levels of spatial tuning and head-direction tuning (with perhaps slightly sharper head-direction tuning in Egyptian fruit bats, but this may have been due to the larger size of the behavioral arena used for this species). We thus conclude, at least based on this standard analysis, that most hippocampal place cells in bat CA1 exhibited significant head-direction tuning.

Next, we tested the uniformity of the preferred head-direction distribution (Fig. $2 F$ ). When pooling together the cells from all five animals, the distribution of the preferred head direction, for all the directionally tuned cells, was not significantly different from uniform (Fig. $2 F$, all data; $\chi^{2}$ test for uniformity: $p=0.37$ ). When analyzing separately the data from the three Egyptian fruit bats, the distributions of the preferred head directions were also not significantly different from uniform-neither when pooling all the neurons from these three bats $\left(\chi^{2}\right.$ test for uniformity: $p=$ 0.48 ), nor for each individual bat (Fig. $2 F$, red, orange, and gray colors; $\chi^{2}$ test: $p>0.4$ for each of these individual bats). However, this was not the case for the big brown bats. In those two 


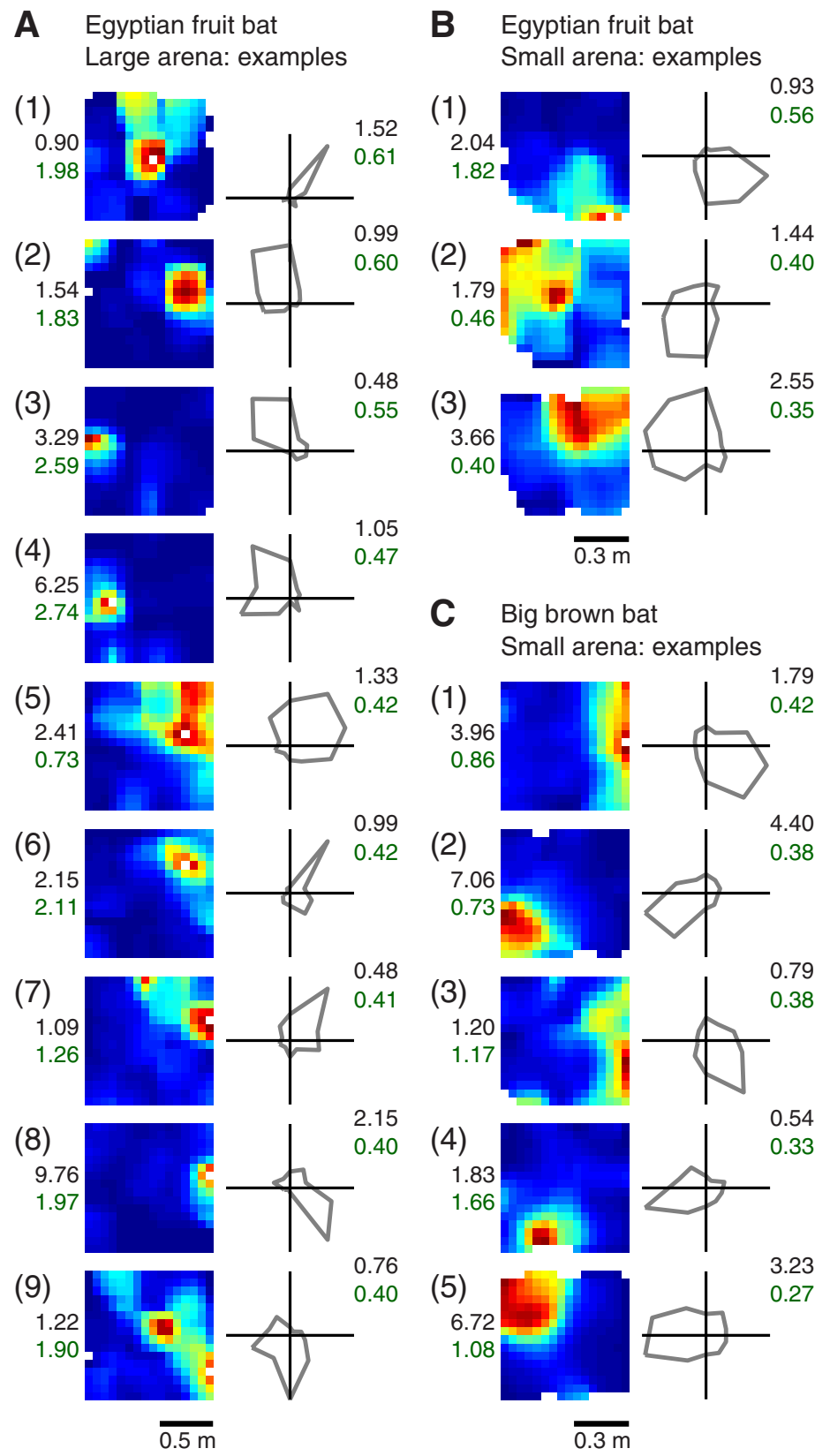

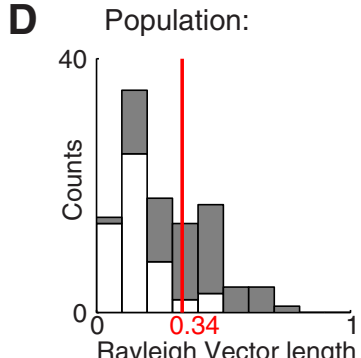

E
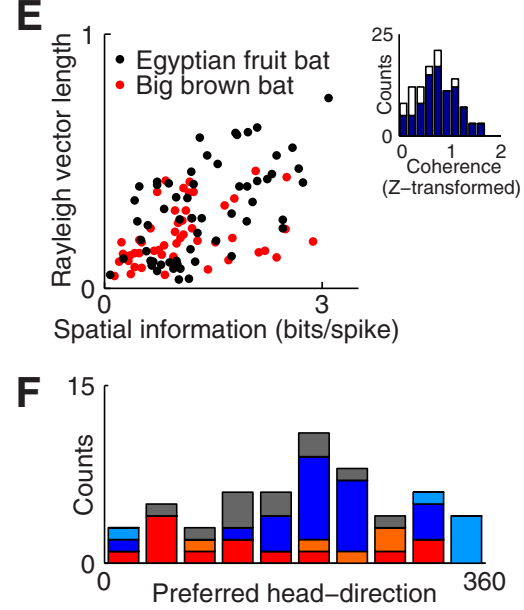

G

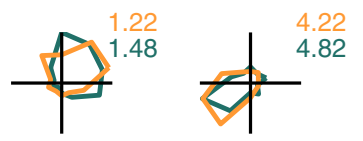

H

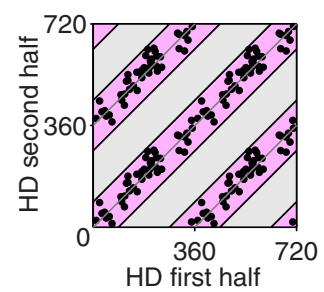

I

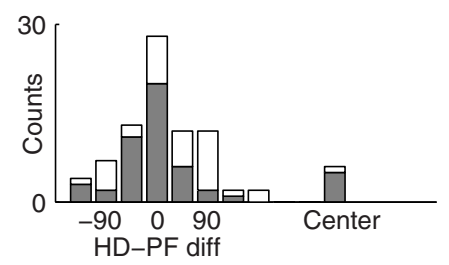

Figure 2. Hippocampal CA1 neurons in two bat species exhibit head-direction selectivity during crawling behavior. $\boldsymbol{A}-\boldsymbol{C}$, Examples of spatial firing-rate maps (left) and head-direction tuning curves (right) for nine hippocampal neurons recorded from Egyptian fruit bats in the large arena $(\boldsymbol{A})$, three neurons in the small arena $(\boldsymbol{B})$, and five neurons from big brown bats crawling in a small arena (C). Color scale: firing rate, with blue indicating zero firing and red maximal firing rate (indicated by numbers in black). Green numbers, spatial information (left, spatial maps) or Rayleigh vector length (right, head-direction curves). Note that many of the hippocampal neurons showed strong head-direction tuning, in addition to the classical place tuning. $\boldsymbol{D}$, Population histogram of Rayleigh vector lengths for all the $n=108$ behaviorally active neurons (not necessarily place cells), from both bat species, showing separately the neurons with significant Rayleigh vector length (according to the single-cell shuffling criterion, see Materials and Methods; gray bars, $n=56$ cells) and nonsignificant neurons (white bars, $n=52$ cells). Red line, 95 th percentile of the shuffling for the entire population. $E$, Population scatter plot of Rayleigh vector length versus spatial information, for all the behaviorally active cells from Egyptian fruit bats (black dots; $n=58$ cells, pooling data from cells recorded in large and small arenas) and big brown bats (red dots; $n=50$ ). The correlation between the two variables could reflect a genuine correlation between spatial and directional tuning, or it could reflect a byproduct of behavioral correlations (see main text). Note that the neuronal population does not cluster into two separate subpopulations of place cells versus head-direction cells. Inset, Histogram of Z-transformed coherence values of the firing-rate maps, for all these $n=108$ neurons (blue bars; $n=86$ neurons with significant spatial information, according to the $95 \%$ shuffling criterion, see Materials and Methods; white bars, nonsignificant cells). $\boldsymbol{F}$, Distribution of preferred head directions for all the cells of each individual bat (colors). Gray color, Egyptian fruit bat small arena; red and orange, Egyptian fruit bats large arena; and dark and light blue, big brown bats. $G$, Examples of stability of the head-direction tuning curve between the first half of the behavioral session (dark turquoise) and the second half (light orange). $\boldsymbol{H}$, Population analysis for all the neurons with significant directional tuning that also had $\geq 50$ spikes in each of the two halves of the session (dots; $n=46$ cells). This scatter plot shows that the preferred head direction was stable between the two halves of the session (all dots fall along the diagonal identity line). Both axes are plotted from 0 to $720^{\circ}$, repeating each angle twice; pink area, region where head-direction differences between the first and second halves of the session are smaller than $\pm 90^{\circ}$. I, Distribution of the angular differences between the preferred head direction (HD), and the direction of the wall closest to the place field (PF; the latter direction was computed by dividing the arena into 9 square regions, comprised of the 8 cardinal directions and the Center square, and determining in which of these 9 regions was the location of the highest firing rate of the neuron). Gray and white colors: cells with significant and nonsignificant head-direction tuning, respectively. $\mathrm{HD}$, head direction; PF, place field. 
bats, the distribution was significantly different from uniform $\left(\chi^{2}\right.$ test for uniformity: $p=0.01$ when pooling data from both animals, and $p<0.01$ for each of the individual bats), with an over-representation around 0 and $180^{\circ}$ (Fig. $2 F$, dark blue and light blue). This asymmetry in big brown bats might possibly arise from the presence of prominent landmarks at 0 and $180^{\circ}$ in that arena (Ulanovsky and Moss, 2007). We note that simultaneously recorded cells were not more similar in their preferred head direction than pairs of neurons recorded from the same bat on different days ( $p>0.5$, shuffle test); additionally, the difference between simultaneously recorded cells did not differ significantly from a uniform distribution ( $\chi^{2}$ test for uniformity: $p>0.25$ ).

Finally, we asked whether the headdirection tuning of these neurons was stable across time. We computed the head-direction tuning curve for the first half versus the second half of the behavioral session, and found that most individual CA1 neurons indeed exhibited stable tuning between the two halves (Fig. $2 G$, examples; turquoise and orange colors represent the first and second half, respectively). Population analysis confirmed that, for the large majority of neurons, the preferred direction remained the same between the two halves of the session (Fig. $2 \mathrm{H}$, most dots fall along the diagonal). We thus conclude that the head-direction tuning of bat hippocampal CA1 neurons was stable, at least over the timescale of one recording session.

\section{Hippocampal place cells show head-direction tuning even when controlling for the behavioral coupling between the animal's head direction and position}

The analyses we conducted above (Fig. $2 A-H$ ) did not take into account the possible behavioral coupling between head direction and location, which may bias the results (Muller et al., 1994). For example, because the animal's head can be physically located in a corner of the arena only when the animal is facing toward that corner, then if there is a place field in this corner, a "naive" calculation of the head-direction tuning, similar to the one in Figure 2 , will show that this neuron has a preferred head direction pointing outward, toward the corner, even if this neuron does not have a true head-direction preference at all (i.e., it is omnidirectional). Generally speaking, this type of problem would be most evident for place fields located close to the edges of the arena. This might explain the head-direction tuning curves of cell 5 in Figure $2 A$ or cell 2 in Figure $2 C$, whose head-direction curves are directed toward the corner where the place field is located. However, such explanation would presumably not explain the firing of cell 1 in Figure $2 A$, whose place field is located at the center of the arena, yet it shows a sharp head-direction tuning curve; nor would it seem to explain the firing of cell 2 in Figure $2 A$, where the headdirection curve is pointing away from the corner. Population
B

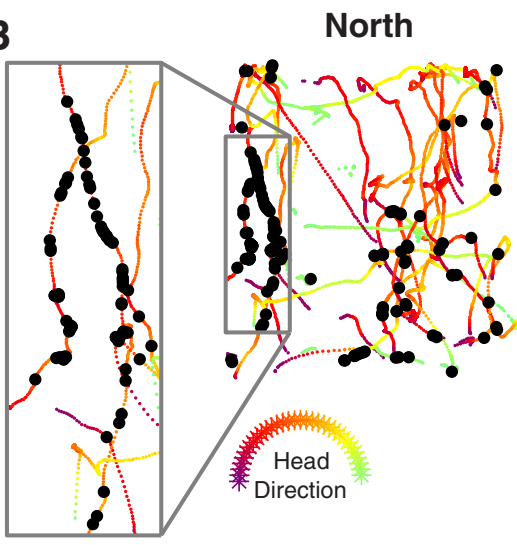

C

South

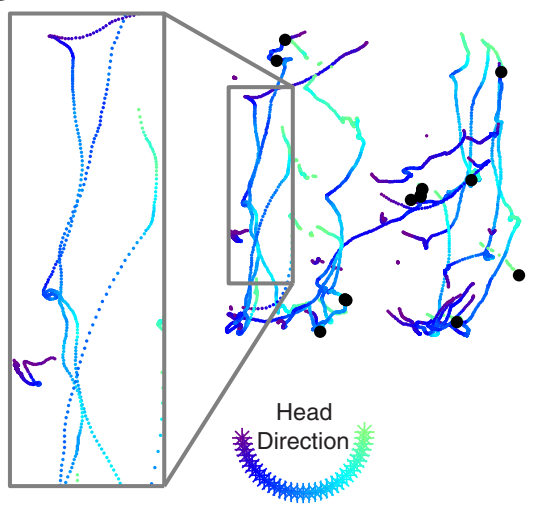

Full data

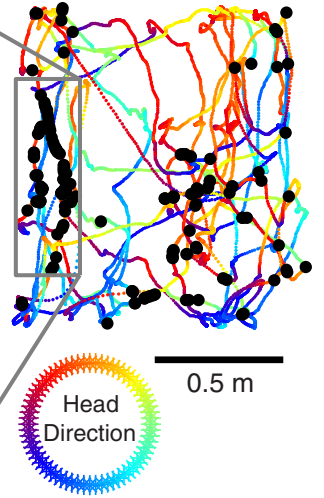

Direction

Figure 3. Example of directional tuning in a single hippocampal CA1 neuron during individual passes through the neuron's . $A$, Full data. Line, bat's crawling trajectory, colored according to the head direction (see color circle); black dots, location northbound passes through the place field ( $\boldsymbol{B}$, inset: red/orange colors), while no spikes were emitted during southbound passes through the place field ( $\boldsymbol{C}$, inset: blue/cyan colors).

analyses supported these observations (Fig. 2I). A histogram of the angular differences between the preferred head direction and the direction to the nearest wall showed that in many neurons, the head-direction tuning pointed toward the wall nearest to the place field (Fig. $2 I$, angular difference $\sim 0^{\circ}$ ); however, a substantial fraction of neurons had an angular difference that was quite different from $0^{\circ}$ (Fig. $2 I$ ). Also, a number of cells had place fields located in the center of the arena (Fig. 2I, "Center"), both of which would not be easily explained by behavioral couplings. Thus, it is clear that a more formal analysis is needed to test whether the empirically observed head-direction tuning curves of hippocampal neurons are indeed a byproduct of their spatial firing (place field), or not. To this end, we would need to decouple the behavioral coupling between head direction and location.

As a first step, we examined the directional firing of neurons within a restricted region of space, thus controlling (at least partially) for the spatial effects, and asked whether hippocampal neurons in a $2 \mathrm{D}$ arena have a head-direction preference within a specific, controlled location. Figure $3 A$ shows an example of a neuron that discharged most of its spikes when the animal was next to the western wall of the arena (and it also discharged some spikes elsewhere). When separating the data into trajectories in which the head direction was pointing north (Fig. $3 B$ ) versus those when the head direction was pointing south (Fig. $3 C$ ), it 
A

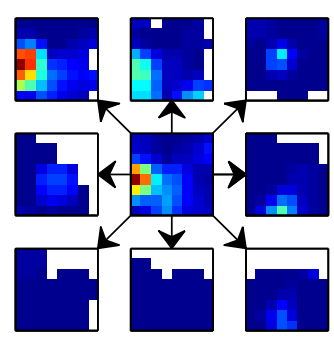

C

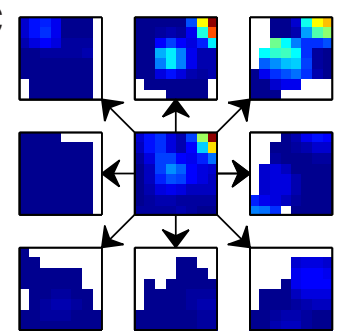

E

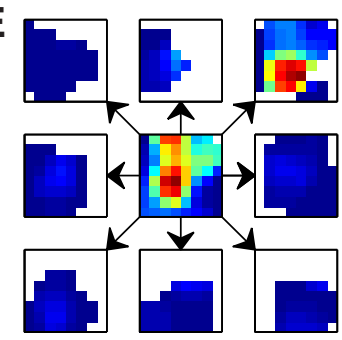

G
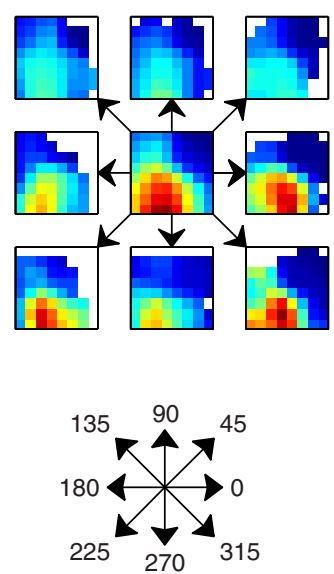
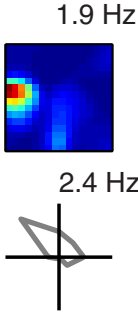

$2.9 \mathrm{~Hz}$

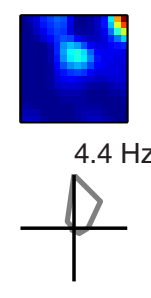

$0.8 \mathrm{~Hz}$

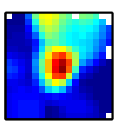

2.6 $\mathrm{Hz}$

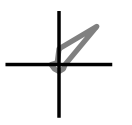

$3.4 \mathrm{~Hz}$

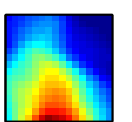

$4.7 \mathrm{~Hz}$

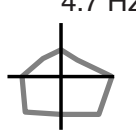

B

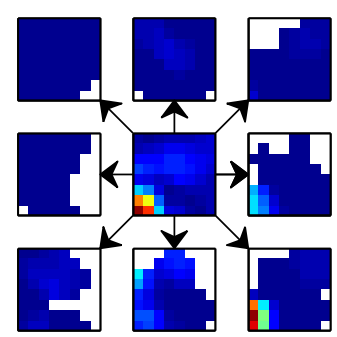

D

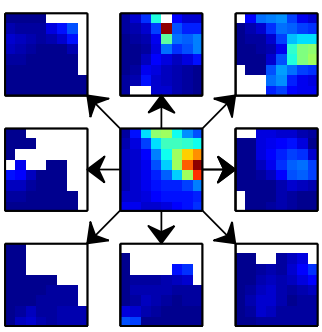

$\mathrm{F}$

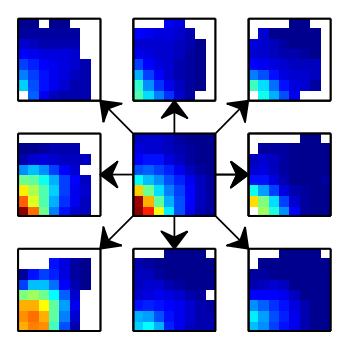

$\mathrm{H}$

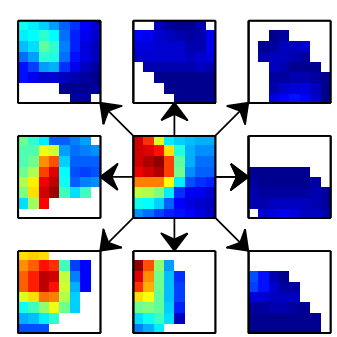

Figure 4. Direction-specific firing-rate maps of bat hippocampal neurons. $\boldsymbol{A}-\boldsymbol{H}$, Eight examples of hippocampal CA1 neurons recorded from Egyptian fruit bats crawling in the large arena $(\boldsymbol{A}-\boldsymbol{E})$, or from big brown bats in small arena $(\boldsymbol{F}, \boldsymbol{G})$, or from Egyptian fruit bats in small arena $(\boldsymbol{H})$. For each neuron, the following plots are shown. Left, firing-rate maps, segmented as a function of the animal's head direction (8 maps for each of the 8 cardinal head directions, $45^{\circ}$ segments; head-direction rosette shown below $\mathbf{G}$; central map shows the average firing-rate map; maps were computed using $8 \times 8$ spatial bins, and smoothed with a Gaussian of width $\sigma=1$ bins and kernel size $3 \times 3$ bins). Top right, Original firing-rate map $(16 \times 16$ spatial bins, smoothed with $\sigma=1.5$ bins and kernel size $5 \times 5$ bins). Bottom right, Head-direction tuning curve. Note that the majority of spatial firing was restricted to a limited directional range.

becomes evident that this cell exhibited highly nonrandom firing across different traversals of the place field. At some traversals many spikes were discharged, but at other traversals through the same area no spikes were discharged, reminiscent of the highly variable firing of rat place cells upon different traversals of place fields (Fenton and Muller, 1998). Importantly, there was a clear relation between the variability in this neuron's in-field firing and the animal's movement direction: during the three traversals
$1.8 \mathrm{~Hz}$

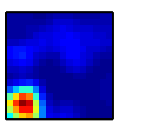

4.6 Hz

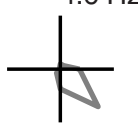

1.1 Hz

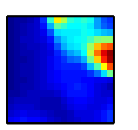

$2.2 \mathrm{~Hz}$

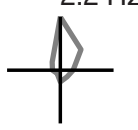

$6.6 \mathrm{~Hz}$

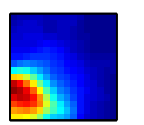

$10.4 \mathrm{~Hz}$

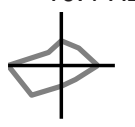

$2.3 \mathrm{~Hz}$

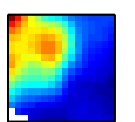

$2.9 \mathrm{~Hz}$

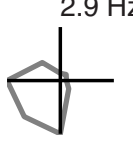

of the place field in which the bat's head direction was pointing north, many spikes were discharged (Fig. 3B, inset, red-colored trajectories, and overlaying spikes = black dots); in contrast, during the three traversals of the place field in which the bat's head direction was pointing south, not a single spike was discharged (Fig. 3C, inset, blue-colored trajectories, no spikes). This type of a bimodal cellular behavior at the same spatial location cannot be explained as a byproduct of place-field firing, as discussed above; instead, it suggests that this neuron truly encoded the animal's head direction (in addition to encoding its position).

We next turned to examine more systematically the firing rates of hippocampal neurons at different directions per specific location. If we assume that place cells in 2D environments are not tuned to head direction, then we would expect that the place field of a neuron will have nearly the same firing rate in all directions, at any given location. To test this assumption, we divided the data into eight headdirection sectors (each sector being $45^{\circ}$ wide) and calculated firing-rate maps separately for each of these eight angular sectors. Figure 4 shows examples of eight neurons, demonstrating that the firing rate was in fact not uniform across different head directions. Although not all the spatial bins were well covered in all the eight head-direction sectors, it is clear that each of these eight example neurons had a preference for a specific head direction, when examining a specific spatial bin (e.g., the bin with the maximal overall firing rate). These examples, showing that the same spatial location can exhibit differences in firing rates that are modulated by the animal's head direction, suggest again that there is true head-direction tuning in hippocampal place cells.

Finally, we asked whether anisotropy in movement velocity could create the observed head-direction tuning. For example, perhaps the animal tends to move faster at a certain direction, and because the firing rates of hippocampal place cells depend on movement velocity (McNaughton et al., 1983), this would create an apparent head directionality at the direction of maximal velocity. To test this possible caveat, we conducted several analyses. First, we compared the preferred head direction of the neurons for fast versus slow movement velocities (by dividing the data into above-median versus below-median velocity), and found that the preferred directions for fast and slow velocities were highly correlated (Fig. $5 A$, middle column, and population summary in $B$; note the clear diagonal band). Second, we compared the preferred head direction of the neurons to the 
A

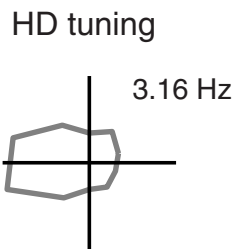

Slow Fast

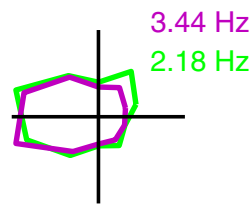

Velocity
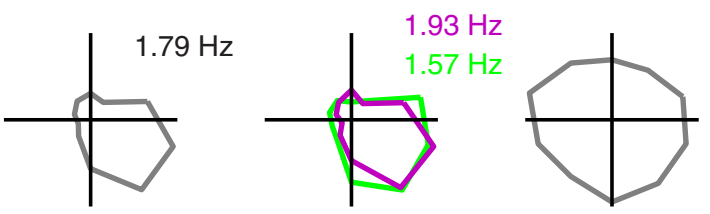

B

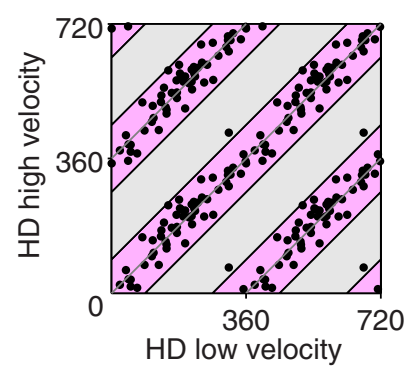

D

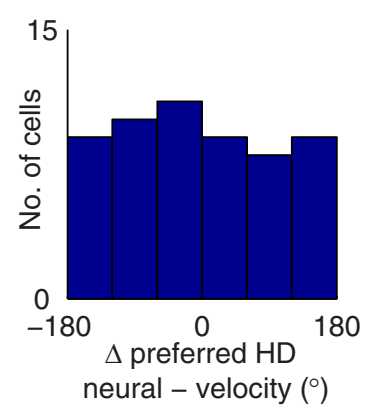

C

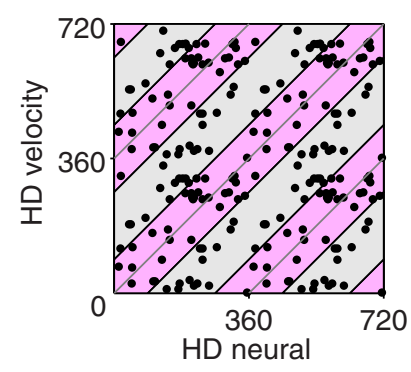

E

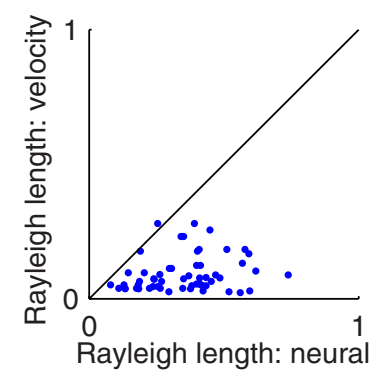

Figure 5. Head directionality is not explained by movement velocity. A, Two example cells (rows), showing the head-direction tuning of their firing (left column), as well as the headdirection tuning split between slow and fast movement velocities (middle column; slow/fast was delineated as below/above the median velocity), and the behavioral directionality of the velocities themselves (right column). HD, head direction. $\boldsymbol{B}$, Similarity between preferred head directions in high-velocity crawling versus low-velocity crawling, plotted for all the 56 neurons with significant head-direction tuning. Dots, neurons. Both axes are plotted from 0 to $720^{\circ}$, repeating each angle twice; pink area, region where head-direction differences between highand low-velocity preferred head directions are smaller than $\pm 90^{\circ}$. C, Comparison of the preferred head-direction tuning of the neurons ( $x$-axis) versus the directional tuning of the velocity in the same session ( $y$-axis). Same color notation as in $\boldsymbol{B}$. $\boldsymbol{D}$, Population histogram of the differences between preferred head direction ("neural") and preferred directional tuning of the velocity ("velocity"). Note the flat distribution. $\boldsymbol{E}$, Comparison of Rayleigh vector length of the head-direction tuning of the neurons ( $x$-axis) and the Rayleigh vector length for the velocity ( $y$-axis). Note that almost all dots (neurons) were below the diagonal, indicating very weak directional tuning of the velocity.

direction of maximal movement velocity, and found that the two directions were in fact not related (Fig. $5 C$, note lack of diagonal band; $D$, note the flat (uniform) distribution of the differences between these two directions). Finally, we compared the directional tuning curve of the neurons (which was quantified by the Rayleigh vector length for the neural tuning curves) versus the velocity directional tuning (which was quantified by the Rayleigh vector length of the velocity-tuning vector). We found that the velocities were in fact much less directionally tuned than the

neurons, and often were not tuned at all (Fig. 5E, nearly all dots are below the diagonal; see also examples of omnidirectional tuning of velocities in $A$, right, which are quite different from the clearly directional neural tuning curves, left). Together, these analyses suggest that the velocity was not directionally tuned and could not underlie the head-direction tuning of hippocampal neurons. Instead, hippocampal place cells seem to exhibit genuine head-direction tuning.

Hippocampal place cells carry head-direction information in the "spontaneous spikes" outside their place field, suggesting that these are not truly "noise spikes"

After demonstrating in Figure 4 that the firing of hippocampal CA1 neurons can be modulated by head direction, at a given location, we now turned to examine the complementary question: whether a hippocampal neuron can be tuned for the same head direction in different spatial positions. Specifically, we asked whether, in addition to head-direction tuning within the place field (as shown in Fig. 4), there could be a head-direction tuning in the sparse "spontaneous" firing of spikes outside of the place field (which are often referred to as noise spikes). To answer this question, we first analyzed explicitly the head-direction tuning inside versus outside the place field, for all the place cells that had only a single field ( $n=60$ neurons; see Materials and Methods). Figure $6 A$ shows three example cells (rows) for which we separated the data into nonoverlapping in-field and out-field parts of the arena, and calculated separately for each the head-direction tuning of the cell. These examples show that the preferred direction of each of these cells was quite similar between the in-field and out-field parts of the firing-rate map (Fig. $6 \mathrm{~A}$, right column, compare red curves and blue curves; note that, by definition, the firing rate inside the place field was always higher than outside it).

Population analyses confirmed that, indeed, a large fraction of neurons showed similar head-direction tuning inside and outside of the place field. First, Rayleigh vector lengths for head-direction tuning, based on the out-field firing, were significant for a substantial fraction of the place cells (Fig. 6B; 21/54 neurons were significantly tuned based on cell-specific shuffling, gray bars; we included here only those place cells that emitted $>50$ spikes outfield: $n=54$ neurons; see Materials and Methods). Note that the significance threshold for the Rayleigh vector length of the outof-field activity (0.32; Fig. $6 \mathrm{~B})$ was very similar to the significance threshold for the full data (0.34; Fig. 2D). Second, population analysis showed that there is indeed a high similarity between the preferred head direction in-field and out-of-field, across the hippocampal neural population. When plotting the preferred head direction out-field versus in-field, most neurons were located along the diagonal (identity line; Fig. 6C; more neurons inside pink area than inside gray area). When plotting a histogram of the differences in head direction between in-field and out-field data, most neurons were closer than chance to a $0^{\circ}$ difference (Fig. $6 D$; $43 / 60$ or $72 \%$ of the neurons were inside the pink area which denotes the area where the in-field and out-field angles differ by $<90^{\circ}$, sign test, $p<0.001$; we included in this analysis only those place cells that had a single field and good behavioral coverage both in-field and out-field: $n=60$ neurons; see Materials and Methods). Further, if we assume a null hypothesis of a random uniform distribution in Figure $6 D$, then we expect by chance to have a mean absolute difference of $90^{\circ}$ between random preferred directions, while we observed in fact a significantly lower difference (mean absolute difference: $67.3^{\circ} ; z$-test, $p<0.01$ ); additionally, the angular difference distribution was significantly nonuniform $\left(\chi^{2}\right.$ test: $\left.\chi^{2}=17.7, \mathrm{df}=8, p<0.05\right)$. Similar results 
A
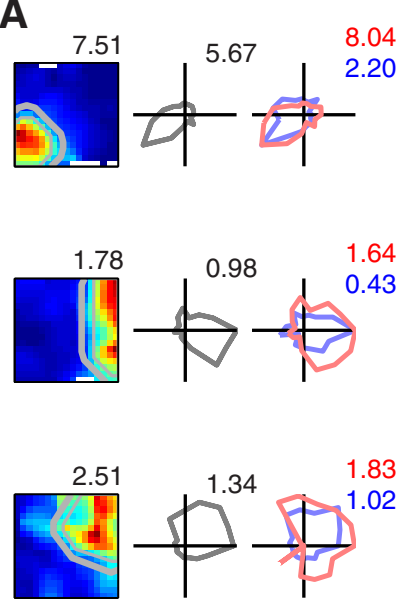
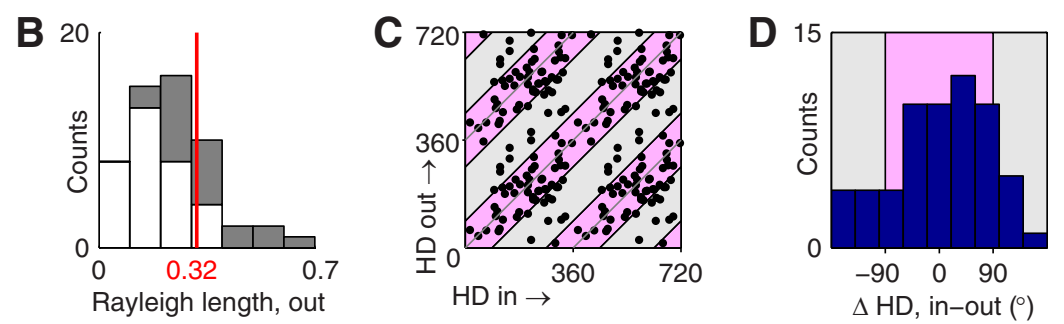

E

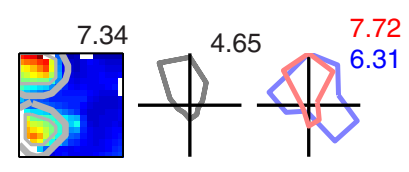

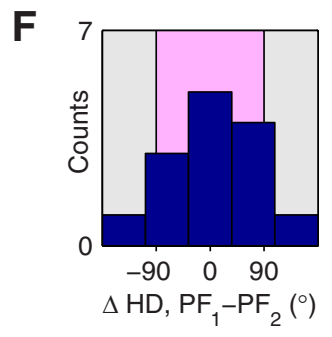

Figure 6. Head-direction tuning inside and outside of the place field. $\boldsymbol{A}$, Three example neurons (rows). Left column, Spatial firing-rate map, with the firing field delineated. Thin gray line, place-field delineation; thick gray line, two-bin margin around the place field (see Materials and Methods). Middle column, Head-direction tuning curve. Right column, Head-direction tuning curve inside the place field (red line) and outside of the place field (blue line), with peak firing rates indicated with matching colors. $\boldsymbol{B}$, Population histogram of Rayleigh vector length, calculated for out-of-field data only; same notation as in Figure 2D.C, Population scatter plot showing the out-field preferred head direction (HD out; $y$-axis) versus in-field preferred head direction (HD in; $x$-axis). Dots, neurons. Both axes are plotted from 0 to $720^{\circ}$, repeating each angle twice. Pink area, region where head-direction differences between in-field and out-field preferred head directions are smaller than $\pm 90^{\circ}$; light-gray area, differences larger than $\pm 90^{\circ}$. Note that most dots were inside the pink region, suggesting that most neurons had similar in-field and out-field preferred head directions. D, Population histogram of the difference between the preferred head direction inside and outside of the place field. Pink and light-gray areas, same notation as in $\boldsymbol{C}$. Analyses in $\boldsymbol{C}$ and $\boldsymbol{D}$ were done over all the place cells that had a single field, and that had valid head-direction data both in-field and out-field ( $n=60$ cells from both bat species; see Materials and Methods). $\boldsymbol{E}$, Example of a neuron with two place fields; similar notation to $\boldsymbol{A}$. The right plot shows the head-direction tuning inside field 1 and field 2 (red and blue lines, respectively). $\boldsymbol{F}$, Population histogram of the differences between preferred head directions for pairs of place fields belonging to the same neuron ( $\mathrm{PF}_{1}$ and $\mathrm{PF}_{2}$ : place fields 1 and 2$)$. Same notation as in $\boldsymbol{D}$.

were obtained also when we included in the analysis only the subset of "classical" place cells-neurons with a single field in their firing-rate map-which met the shuffling criterion, and that, in addition, had spatial information $>0.5$ bits/spike $(n=31$ cells; $24 / 31$ or $77 \%$ of these neurons were inside the pink area, sign test, $p<0.01$; mean absolute difference: $58.7^{\circ}, z$-test, $p<0.01$; angular difference distribution was again significantly nonuniform: $\left.\chi^{2}=20.4, \mathrm{df}=8, p<0.01\right)$. Thus, we conclude that (1) hippocampal CA1 place cells encoded head-direction tuning also in spikes that occurred outside of their place field (Fig. 6B) and (2) the preferred head direction was similar inside and outside the place field (Fig. 6C,D).

Further, we compared the preferred head directions for pairs of place fields belonging to the same cell (for neurons with $\geq 2$ fields). Figure $6 E$ shows an example of a neuron with two fields, and Figure $6 F$ shows population comparison of the preferred head directions between field-pairs-demonstrating a rather similar head-direction tuning across the pairs of fields that belong to the same neuron (Fig. $6 F$; $n=14$ pairs; $10 / 14$ or $71.4 \%$ of these neurons were inside the pink area).

Finally, we went beyond the dichotomous separation between in-field versus out-field, and sought to determine the headdirection tuning at a higher spatial resolution; namely, we asked what is the head-direction tuning at each spatial-position bin. We first examined an existing method, the maximal-likelihood method (Burgess et al., 2005). We created a simulated neuron (Fig. 7, top-cell 1) with a place field at one of the corners, and added a true head-direction tuning to this neuron (peak firing rate of $3 \mathrm{~Hz}$ at the preferred direction, over a $0.3 \mathrm{~Hz}$ baseline, Rayleigh vector length $=0.55)$, and made this preferred direction uniform across the environment (Fig. $7 B$, top, note the spatially uniform blue color; see also Materials and Methods). The maximum-likelihood method indeed reconstructed very well the preferred head direction of this neuron (Fig. 7C, top; the reconstructed head direction, blue color, was very similar to $B$ ). An alternative method that we examined was to calculate the spatial firing-rate map of the neuron, separately for each of the eight head-direction sectors, employing $8 \times 8$ spatial binning, and then computing for each spatial bin its preferred head direction (Fig. 7D, top; see details in Materials and Methods). This method, which we called the "per-spatial-bin approach" (because it estimated the head-direction tuning separately for each spatial bin), yielded reasonable results (mostly bluish colors in Fig. $7 D$, top; see also histogram in Fig. 7E, top), although the reconstruction was more noisy than the maximum-likelihood method (Fig. 7C, top). However, the per-spatial-bin approach was superior to the maximum-likelihood method when we created another simulated neuron that had different preferred head directions inside versus outside its place field (Fig. 7, middle-cell 2): In this case, the maximum-likelihood method failed to capture the differences in preferred head directions across the arena (the reconstruction in Fig. $7 C$, middle, is very different than the original data in Fig. $7 B$ ), because the maximum-likelihood method inherently can estimate only one "average" head-direction value (Fig. $7 C$, middle, the light-blue color, which is in between the two actual preferred head directions of cell 2). In contrast, the perspatial-bin approach captured quite well the spatially heterogeneous head-direction tuning (Fig. $7 D$, middle; exhibits greenish colors everywhere except the blue corner, similar to the original simulation data in Fig. $7 B$ ). Therefore, we chose to analyze the real neuronal data using the per-spatial-bin approach, which is more suitable to answer our key question: namely, are there differences in head-direction tuning between different spatial positions.

After validating the method with simulated data, we first calculated for each neuron the spatial firing-rate map of the neuron, 
A

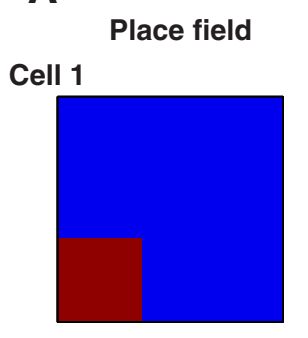

Cell 2

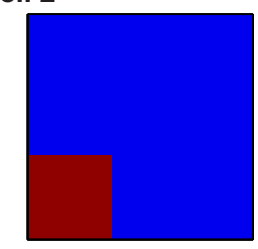

Cell 3

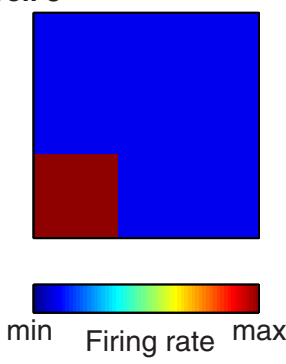

B

Preferred HD

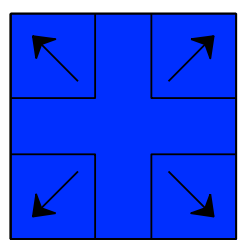

C

HD analysis
max likelihood

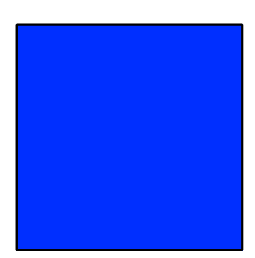

D HD analysis per spatial bins

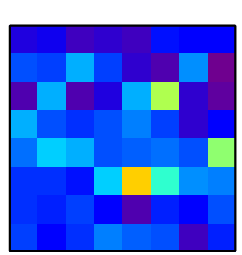

E Location-specific HD histogram

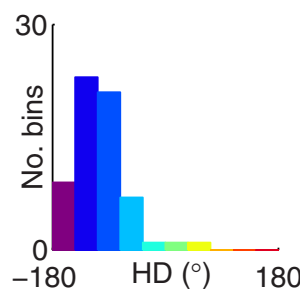

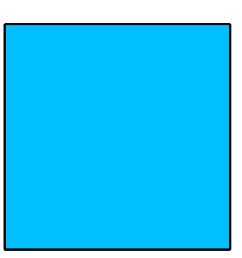
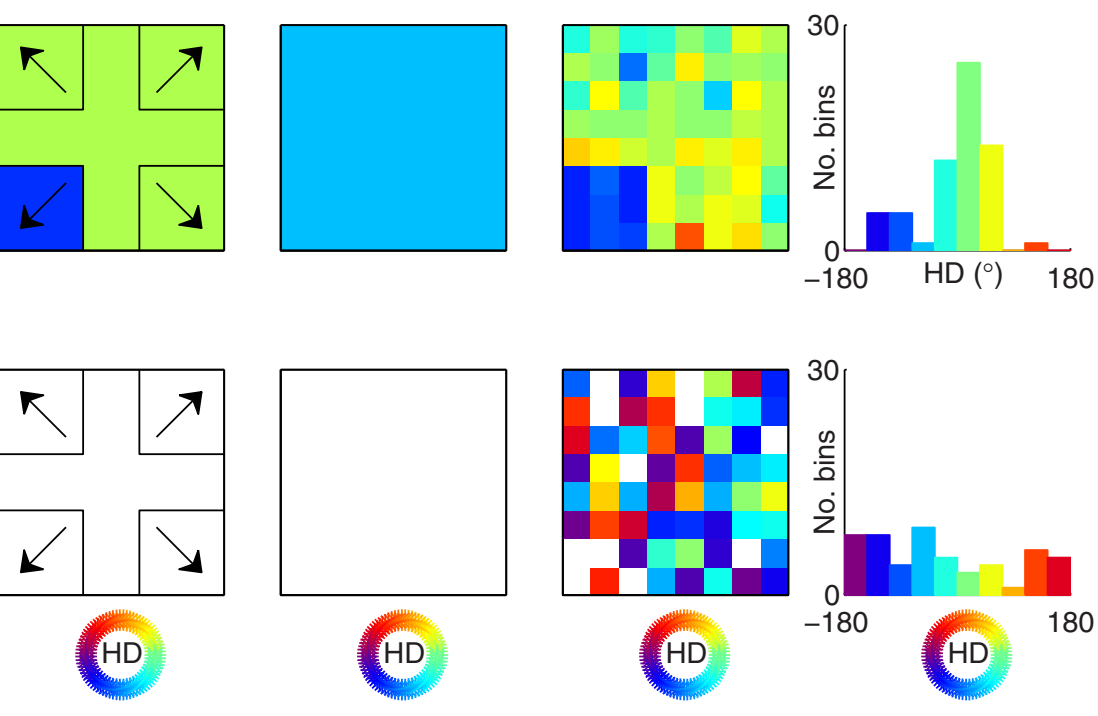

Figure 7. Comparing two methods for estimating the preferred head direction: the maximum-likelihood method, and the location-specific head-direction (HD) map, which are demonstrated here for three simulated neurons (rows). Top (cell 1), A simulated neuron with a place field in its southwest corner, and with a preferred head direction that was uniform throughout the arena. Middle (cell 2), A simulated neuron, with a place field identical to cell 1, but whose head-direction tuning differed comparing in-field versus out-field. Bottom (cell 3), A simulated neuron, with the same place field but no head-direction tuning. $\boldsymbol{A}, \boldsymbol{B}$, The "ground truth" of the simulated data. $\boldsymbol{C}-\boldsymbol{E}$, Head-direction estimates. $\boldsymbol{A}$, Firing-rate maps of each neuron. In all cells, the firing rate in-field was 3 $\mathrm{Hz}$ and out-field $0.3 \mathrm{~Hz}$. B, Preferred head-direction maps. The color of each pixel indicates the preferred head direction at that specific location; see color rosette of preferred head directions at the bottom (white square in third row indicates no head-direction tuning). Arrows show the behavioral bias that we simulated for the animal. In each of the four marked spatial zones (at the four corners), the animal has spent more time facing the indicated direction (fourfold behavioral bias as compared with the opposite direction); in the rest of the arena (" + " zone) the time spent at each direction was distributed uniformly. These behavioral biases were identical for the three neurons. In contrast, the preferred directions of the neural tuning curves differed between these three simulated neurons; namely, cell 1 had a preferred head direction to the southwest across the entire arena ( $\boldsymbol{B}$, top, blue color); cell 2 had a preferred head direction to the southwest (blue) inside its place field, but was tuned to the east direction (green) outside of its place field ( $\boldsymbol{B}$, middle); and cell 3 had no preferred head direction (white; $\boldsymbol{B}$, bottom). $\boldsymbol{C}$, Global estimation of the neuron's preferred head direction, according to the maximum-likelihood method (as described by Burgess et al., 2005). Colors denote the preferred head direction (see rosette); white color indicates there is no head-direction tuning (Rayleigh vector length $<0.2$ ). D, Per-spatial-pixel estimation of the neuron's preferred head direction (head direction estimated separately for each spatial bin; see Materials and Methods). Note that the maximum-likelihood method produces accurate head-direction reconstructions when the preferred head direction is uniform across the space (e.g., in cell 1) but fails to deal with nonuniform preferred head directions (cell 2, note the light-blue color; the estimated preferred direction is in between the two actual preferred directions, blue and green). 0 n the other hand, the per-pixel analysis reconstructed well the spatial distribution of preferred head directions, and was not affected by the behavioral biases (e.g., compare cell $2 \boldsymbol{D}, \boldsymbol{B}$; the reconstructions in column $\boldsymbol{D}$ are not biased by the directions of arrows in $\boldsymbol{B}$ ). See further details in the main text. $\boldsymbol{E}$, Histogram of per-pixel preferred head direction.

separately for each of the eight head-direction sectors, employing $8 \times 8$ spatial binning (Fig. $8 A$; same binning as in Figs. 4, 7). We then computed for each spatial bin its preferred head direction (Fig. 8C) and Rayleigh vector length (Fig. 8D; this computation was done only for spatial bins that were well covered across the various head directions, namely, for spatial bins at which the bat spent at least $300 \mathrm{~ms}$ in each directional sector, for at least seven of the eight head-direction sectors; see Materials and Methods). Examination of the example cells in Figure $8, A-F$ (five cells, rows), suggests that some of the cells seemed to be consistently tuned to the same head direction throughout the arena (Fig. $8 E$; note the narrow histograms of preferred angles over all spatial bins, indicating that the preferred direction of each neuron was highly preserved throughout most of the arena), including far away from the neuron's place field (Fig. $8 F$ ). For example, the first cell in Figure 8, $A-F$ (top row), had nearly the same preferred head direction throughout the arena (Fig. $8 C$, top; note there is red color throughout most of the arena). Next, we calculated for each cell a consistency index, quantifying the consistency of head direction across all the spatial bins (see Materials and Methods). Of those cells that showed similar head-direction tuning across the arena (i.e., neurons above the median line in the histogram of consistency index in Fig. 8G), the majority of neurons exhibited similar head-direction tuning inside their place field and far outside of the place field (Fig. $8 \mathrm{H}$, population head-direction decoherence, red line, is above the chance level denoted by gray horizontal line). Note that while the firing rate decayed rapidly as a function of distance from the place-field center (Fig. $8 \mathrm{H}$, blue line), the head-direction angle decayed much slower (Fig. $8 \mathrm{H}$, red line). In fact, the in-field head direction was preserved, at least to 
A

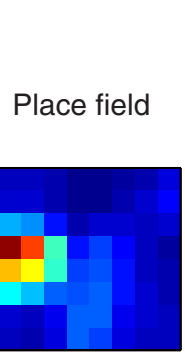

B
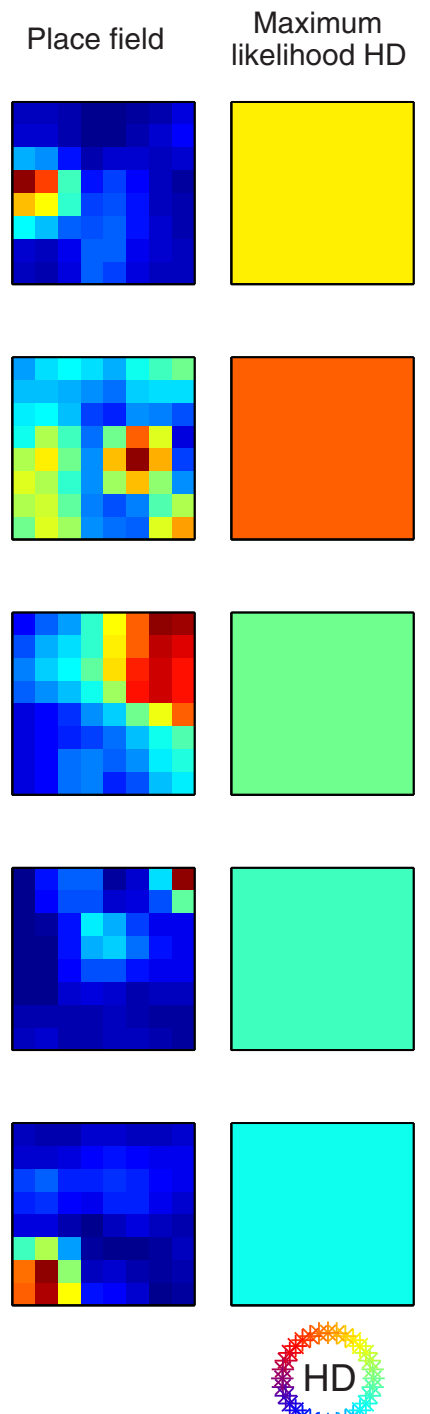

G

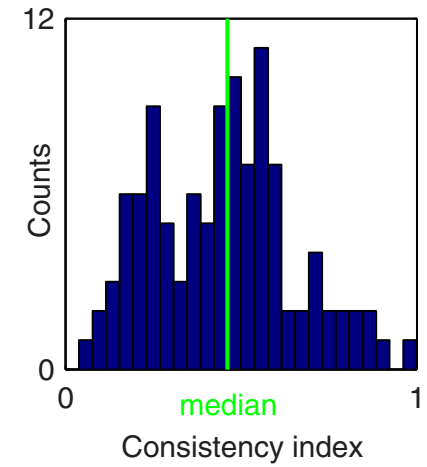

C D

Map of Map of Rayleigh Location-specific preferred HD vector length
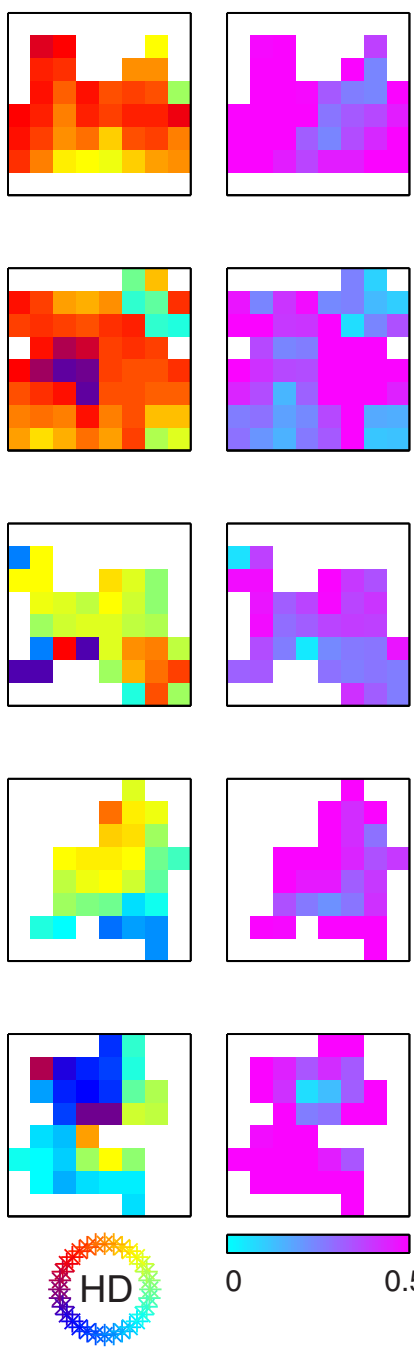
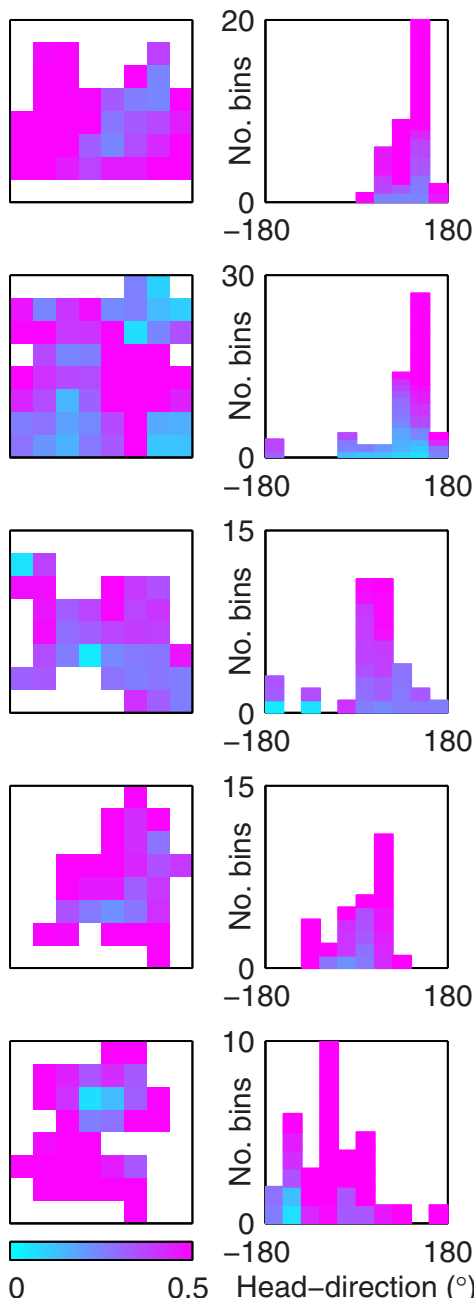

0.5 Head-direction $\left({ }^{\circ}\right)$
$\mathbf{F}$

Decorrelation of FR \& $\mathrm{HD}$ with distance
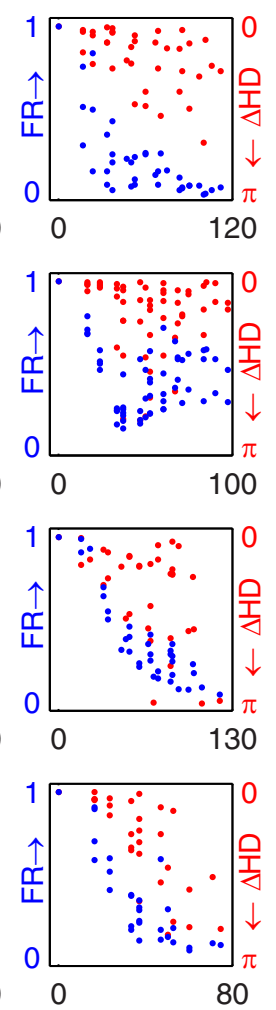

H

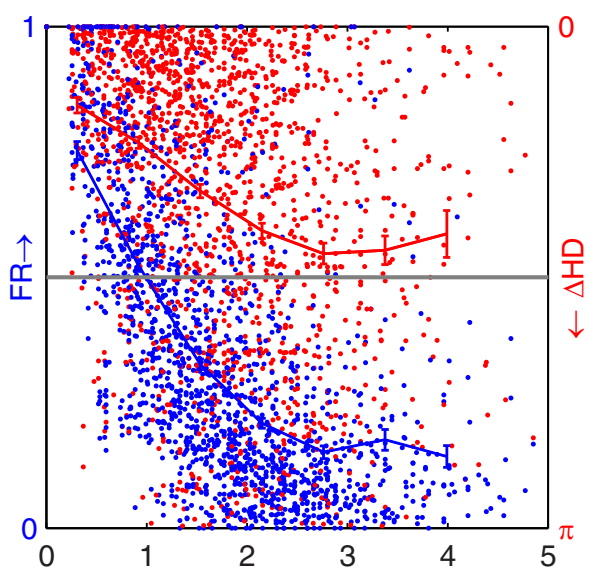

Distance from center of place field (radii)

Figure 8. Location-specific analysis of head-direction tuning. $\boldsymbol{A}-\boldsymbol{F}$, Five example cells (rows). $\boldsymbol{A}$, Spatial firing map (place field, $8 \times 8$ bins, smoothed with a Gaussian of width $\sigma=1$ bins and kernel size $3 \times 3$ bins). $\boldsymbol{B}$, Head-direction estimates according to the maximum-likelihood method (see Materials and Methods). C, Location-specific map of preferred head-direction (HD), computed for each spatial bin that was well covered across the different head directions; colors indicate preferred head direction (see color circle at the bottom). D, Map of Rayleigh vector length values for each spatial bin. Magenta, highly tuned head direction; cyan, poorly tuned (see color-map at the bottom; bins with Rayleigh vector length $>0.5$ were clipped to 0.5 ,(Figure legend continues.) 
some degree, even at very distal locations from the center of the place field-as far as $4-5$ radii of the place field (Fig. $8 H$, red line; see also Materials and Methods). Together, these results suggest that (1) bat hippocampal CA1 neurons showed significant headdirection tuning, quite coherently across the available 2D space and (2) as a consequence, spikes emitted outside of the neuron's place field-which are often referred to as spontaneous or noise spikes-are in fact not just noise, but were tuned to head direction.

Bat hippocampal CA1 neurons are more place cells than they are head-direction cells

To compare the relative saliency of place coding versus headdirection coding in bat hippocampal CA1 cells, we mutually reconstructed the two firing patterns from each other. Namely, we performed an analysis similar to the one described by Muller et al. (1994), whereby we first assumed that hippocampal neurons are pure place cells, which are not tuned to head direction, and then we attempted to reconstruct the observed head-direction tuning curves of hippocampal cells based on their spatial place-field firing alone (Fig. 9A). Note that here, unlike in the other analyses in this study, we used $6 \times 6$ spatial bins and 36 head-direction bins, to keep the same number of bins for the two cases (see Materials and Methods). When conducting this reconstruction analysis, we found that, for many cells, the apparent head-direction tuning could indeed be fully explained by assuming a pure place field, taking into account the empirically observed behavioral coupling between the animal's place and head direction. For example, the first neuron in Figure 9A (top row) exhibited head-direction tuning toward the corner where its place field was located, and this was reconstructed quite well based on the spatial firing-rate map alone (Fig. 9A, top; reconstructed tuning curve on the right matches the original tuning curve on the left). Similar match was exhibited by the third and fifth example neurons in Figure 9A (see value of correlation coefficient below each neuron). Population analysis showed that, at the population level, the large majority of neurons had positive correlations between their original headdirection tuning curves and the head-direction tuning curves reconstructed based on spatial firing alone (Fig. 9C; 98\%, or 106/ 108 of the neurons, had positive correlation coefficient along the $x$-axis, $r\left(\mathrm{HD}, \mathrm{HD}_{\text {est }}\right)>0$; sign test, $\left.p<10^{-28}\right)$.

However, one can also perform the converse analysis: to assume that some hippocampal neurons might in fact be pure head-direction cells, and then try to reconstruct their spatial fir-

$\leftarrow$

(Figure legend continued.) for display purposes only). $\boldsymbol{E}$, Histogram of preferred head directions across all the spatial bins of each neuron, colored according to the Rayleigh vector length for that bin (i.e., the plots in $\boldsymbol{E}$ show the histograms of the directional data from column $\boldsymbol{C}$, colored according to column $\boldsymbol{D}$ ). $\boldsymbol{F}$, Plots showing the firing rate (FR) normalized to maximal rate (blue dots, left $y$-axis), and the changes in head-direction angle compared with the preferred angle at the center of the place field (red dots, right $y$-axis), both plotted versus the distance from the center of the place field ( $x$-axis; see Materials and Methods). $G$, Population histogram of the consistency index (see Materials and Methods). Green line, median value of the distribution. $\boldsymbol{H}$, Population graph showing the decay of the firing rates (blue) and changes in preferred head directions (red) as a function of distance from the place-field center, for all the spatial bins of all the place cells with a consistency index above the median (i.e., above the green line in $\mathbf{G}$; $n=47$ cells). Distance from center of place field ( $x$-axis) was normalized to represent units of place-field radius (see Materials and Methods). Red line, binned averages of the red dots; mean \pm SEM. Note that while the firing rates decayed rapidly as a function of distance from the place-field center, the preferred directions decayed much slower with distance, and remained tuned throughout the arena (red error bars $>$ chance level indicated by gray line). This means that a given neuron tends to represent the same head direction throughout the arena, both inside the place field and outside of it, so the extra-field noise spikes in fact carry a headdirection signal.
A

\section{A}

HD tuning curve original reconstructed

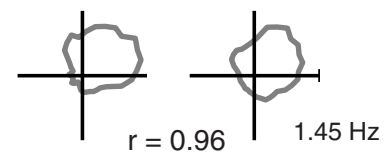

B Place field original reconstructed

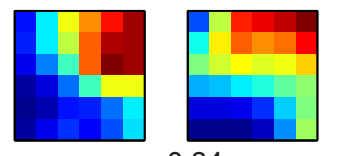

$r=0.84$
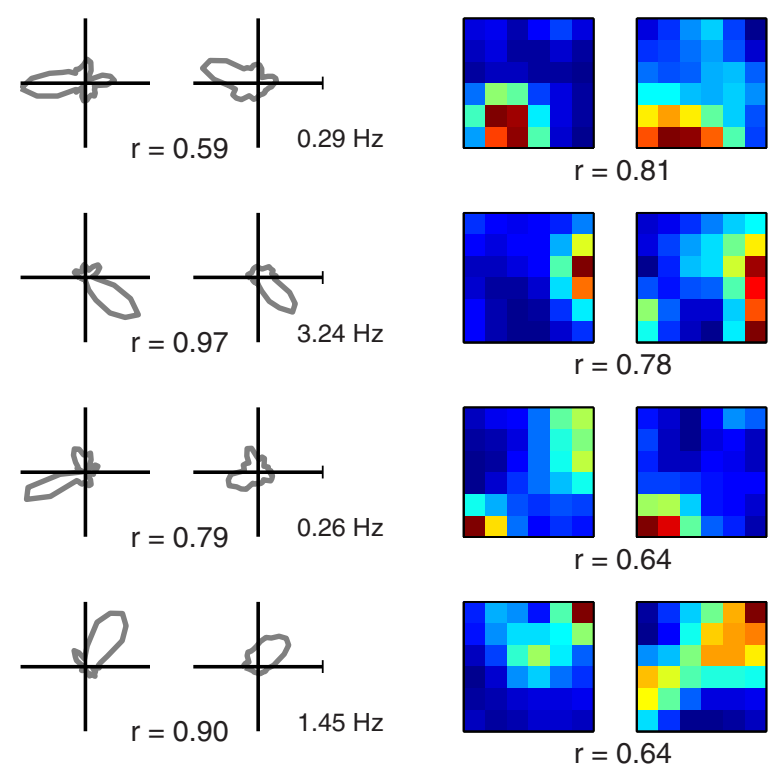

C
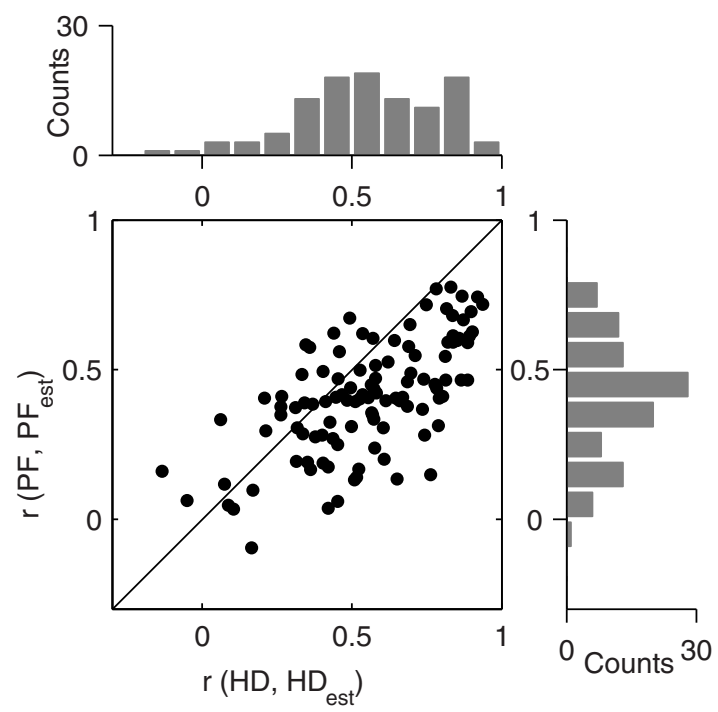

Figure 9. Reconstruction of head-direction (HD) tuning from the spatial firing-rate map, and vice versa. $A$, Examples of five neurons (rows) showing the original head-direction tuning curve (left) and reconstructed curve (right), with reconstruction based on the spatial firing-rate maps (see Materials and Methods). $\boldsymbol{B}$, Examples of the same five neurons as in $\boldsymbol{A}$ (rows), showing the original spatial firing-rate maps (left) and reconstructed maps (right), with reconstruction based on the head-direction tuning curves. Nearest-neighbor smoothing was applied on the tuning curves in $\boldsymbol{A}$ and the firing-rate maps in $\boldsymbol{B}$, for display purposes only (the reconstruction itself was done without any smoothing). $C$, Population scatter plot of the Pearson correlation between the original and reconstructed spatial firing-rate maps ( $y$-axis) versus the Pearson correlation between the original and reconstructed head-directional tuning curves ( $x$-axis); $n=108$ cells. Histograms show the two marginal distributions. PF, place field.

ing (place field) from their pure head-direction tuning curves, and from the behavioral coupling between location and head direction. This converse analysis showed that many place fields can in fact be reconstructed based purely on the head-direction 
tuning of the cells: This was evident in specific examples (Fig. 9B, rows; there is reasonable similarity between reconstructed and original place fields) as well as in population analysis, where positive correlations were found between the shapes of original and reconstructed place fields (Fig. 9C; 99\% of the neurons, 107/108, had positive correlation coefficient along the $y$-axis, $r(\mathrm{PF}$, $\left.\mathrm{PF}_{\text {est }}\right)>0$; sign test, $\left.p<10^{-30}\right)$. Nevertheless, $80 \%(86 / 108)$ of the neurons in Figure $9 C$ were located below the diagonal, indicating that the reconstruction of head-direction curves assuming pure place tuning was better than the converse reconstruction of place fields assuming pure head-direction tuning. In other words, the firing of most hippocampal CA1 neurons was better explained as being place cells than by their head-direction tuning. These results are in agreement with the fact that the fraction of CA1 cells with significantly high spatial information $(80 \%, 86 /$ 108 ) was larger than the fraction of cells with significantly high Rayleigh vector length (52\%, 56/108; both significances were assessed by the shuffling procedure described in Materials and Methods). We therefore conclude that (1) hippocampal neurons are more place cells than they are head-direction cells, which is consistent with established notions on the spatial correlates of hippocampal CA1 neuronal firing in rodents and bats (O'Keefe, 1976; Wilson and McNaughton, 1993; Wood et al., 2000; Lee et al., 2004; Leutgeb et al., 2004; Ulanovsky and Moss, 2007, 2011; Yartsev et al., 2011) and (2) nevertheless, these data also suggest that hippocampal neurons do exhibit true head-direction tuning, which at least partially can account for the observed place-related firing.

\section{Discussion}

We recorded here the activity of single neurons from hippocampal area CA1 of two evolutionarily distant bat species, which crawled in three different open-field arenas. We found that many of the hippocampal place cells, in both bat species, were sensitive not only to the spatial position of the animal in the environment, as expected from the classical place-field concept (O'Keefe and Nadel, 1978; Ulanovsky and Moss, 2007), but also to the head direction of the animal. This finding, that bat hippocampal CA1 neurons can multiplex place $\times$ direction information, is inconsistent with the prevailing view, which posits that during random foraging in $2 \mathrm{D}$ open-field environments, hippocampal place cells are generally not sensitive to the animal's head direction (Andersen et al., 2007). Further, we found here that the head-direction signal was, in many cases, maintained also outside of the neuron's firing field. This suggests a surprising new functional role for the noise spikes emitted outside of the neuron's place field; namely, that these spontaneous spikes in fact carry head-direction information.

Coding for the animal's head direction is considered a pivotal component of the brain's navigation system, and has been reported in many of the structures implicated in spatial navigation, such as the medial entorhinal cortex, presubiculum and parasubiculum, thalamic nuclei, and additional adjacent regions (Taube, 2007). Interestingly, in the hippocampus proper, there have been nearly no reports on coding for the animal's head direction when animals move randomly in open-field environments; instead, truly directional firing was reported only for running on 1D tracks (McNaughton et al., 1983; Frank et al., 2001; Kjelstrup et al., 2008). Here, we initially used standard analysis techniques that are classically used to assess head-direction tuning in other brain structures, and found that many of the hippocampal place cells showed tuning to head direction. We then introduced analysis methods (Figs. 6-8), which allowed us to demonstrate that this head-direction tuning was real, and was not a byproduct of correlations between behavioral variables (i.e., behavioral coupling between position and head direction). Encoding of spatial and directional information was not mutually exclusive, and in many cases these two codes coexisted in the same neuron. These results suggest that hippocampal place cells carry both a spatial and a directional signal, similar to the spatial $\times$ directional signals carried by "conjunctive grid cells" in the medial entorhinal cortex of rats (Sargolini et al., 2006) and bats (Yartsev et al., 2011), or the spatial $\times$ directional signals carried by "place-by-direction cells" in the subiculum (Cacucci et al., 2004). Our results suggest, however, that such spatial $\times$ directional conjunctive signals are carried also by neurons located inside hippocampus proper, in dorsal CA1.

While hippocampal place cells fire mainly within a confined region of the available environment, these neurons often emit spikes (although at much lower firing rates) also in locations outside of the neuron's firing-field. Here we found that these noise spikes carried a strong head-direction signal, which in many cases resembled remarkably the in-field directional coding (Fig. 6). This finding further supports the notion that there is a real head-direction tuning in hippocampal CA1 neurons; it also suggests that, surprisingly, out-field noise spikes may in fact be functionally useful, by carrying directional information.

Could these results be explained by the bat's CA1 neurons being "spatial-view cells," similar to those reported in monkeys (Georges-François et al., 1999)? Although we have reported in the past some evidence for possible (weak) spatial-view tuning in bat CA1 (Ulanovsky and Moss, 2011), the current results argue against this interpretation, because we have found here that the head-direction tuning is often quite uniform across the arena (Fig. 8), which is not consistent with a spatial-view tuning, under which the preferred head direction should be changing in a "pinwheel-like" manner across the arena, always pointing toward a certain location on one of the walls. We never observed such pinwheel arrangements of head-direction tuning. Therefore, we believe that our results are most consistent with the notion that these neurons are place cells, which also exhibit a true modulation by head direction.

What are the possible explanations for the apparent difference between our results on spatial $\times$ directional tuning in bat CA1 cells, versus the previously published study, which reported that rat CA1 cells exhibit only spatial, but no directional tuning (Muller et al., 1994)? One possible difference could be in the training or environment. However, the environments we used were constructed explicitly to mimic as closely as possible the typical rodent open-field environments, including a square shape, walls, and prominent landmarks (Ulanovsky and Moss, 2007) or a large cue-card (Yartsev et al., 2011). The training for a random "food chasing" task was also very similar to that used in rats. Therefore, the training/task/environment does not seem to explain these differences. A second possibility is that species differences are the underlying cause; for example, because bats mainly rely on distal and directed sensory systems (vision, echolocation) as compared with the more proximal and omnidirectional primary senses of rats (olfaction, whisking). Last, the difference could be due to the analytical techniques: the new analyses that we introduced here, and applied on bat place cells (Figs. 6, 8), were not conducted so far on rodent hippocampal place cells. Further, in Figure 8, $E$ and $F$, we demonstrated that these analysis methods can reveal head-direction sensitivities that are not revealed by the methods that were previously applied to rat data (Burgess et al., 2005). These new analyses could therefore 
be used in the future also on data collected from rats running in open-field arenas. We speculate that such analyses in rats might reveal that hippocampal place cells in rats may also carry true head-direction information-similar to our results in bats.

Finally, these findings suggest that bat hippocampal neurons conjunctively encode both map and compass information, which is in line with the classical map and compass theory of navigation (Kramer, 1953). Hence, surprisingly, the hippocampus may be implementing not only a "cognitive map," but seems to contain a neural implementation of a map and compass.

\section{References}

Andersen P, Morris RG, Amaral DG, Bliss T, O'Keefe J (2007) The Hippocampus Book. New York: Oxford UP.

Barry C, Hayman R, Burgess N, Jeffery KJ (2007) Experience-dependent rescaling of entorhinal grids. Nat Neurosci 10:682-684. CrossRef Medline

Boccara CN, Sargolini F, Thoresen VH, Solstad T, Witter MP, Moser EI, Moser M-B (2010) Grid cells in pre- and parasubiculum. Nat Neurosci 13:987-994. Medline

Burgess N, Cacucci F, Lever C, O'keefe J (2005) Characterizing multiple independent behavioral correlates of cell firing in freely moving animals. Hippocampus 15:149-153. CrossRef Medline

Cacucci F, Lever C, Wills TJ, Burgess N, O'Keefe J (2004) Theta-modulated place-by-direction cells in the hippocampal formation in the rat. J Neurosci 24:8265-8277. CrossRef Medline

Eichenbaum H, Wiener SI, Shapiro ML, Cohen NJ (1989) The organization of spatial coding in the hippocampus: a study of neural ensemble activity. J Neurosci 9:2764-2775. Medline

Fenton AA, Muller RU (1998) Place cell discharge is extremely variable during individual passes of the rat through the firing field. Proc Natl Acad Sci U S A 95:3182-3187. CrossRef Medline

Frank LM, Brown EN, Wilson MA (2001) A comparison of the firing properties of putative excitatory and inhibitory neurons from CA1 and the entorhinal cortex. J Neurophysiol 86:2029-2040. Medline

Georges-François P, Rolls ET, Robertson RG (1999) Spatial view cells in the primate hippocampus: allocentric view not head direction or eye position of place. Cereb Cortex 9:197-212. CrossRef Medline

Hafting T, Fyhn M, Molden S, Moser M-B, Moser EI (2005) Microstructure of a spatial map in the entorhinal cortex. Nature 436:801-806. CrossRef Medline

Hargreaves EL, Yoganarasimha D, Knierim JJ (2007) Cohesiveness of spatial and directional representations recorded from neural ensembles in the anterior thalamus, parasubiculum, medial entorhinal cortex, and hippocampus. Hippocampus 17:826-841. CrossRef Medline

Kjelstrup KB, Solstad T, Brun VH, Hafting T, Leutgeb S, Witter MP, Moser EI, Moser M-B (2008) Finite scale of spatial representation in the hippocampus. Science 321:140-143. CrossRef Medline

Kramer G (1953) Die Sonnenorientierung der Vögel. Verh Dtsch Zool Ges $1: 77-84$.

Langston RF, Ainge JA, Couey JJ, Canto CB, Bjerknes TL, Witter MP, Moser EI, Moser M-B (2010) Development of the spatial representation system in the rat. Science 328:1576-1580. CrossRef Medline

Lee I, Yoganarasimha D, Rao G, Knierim JJ (2004) Comparison of popula- tion coherence of place cells in hippocampal subfields CA1 and CA3. Nature 430:456-459. CrossRef Medline

Leutgeb S, Leutgeb JK, Treves A, Moser M-B, Moser EI (2004) Distinct ensemble codes in hippocampal areas CA3 and CA1. Science 305:12951298. CrossRef Medline

McNaughton BL, Barnes CA, O'Keefe J (1983) The contributions of position, direction, and velocity to single unit activity in the hippocampus of freely-moving rats. Exp Brain Res 52:41-49. Medline

Moser EI, Kropff E, Moser M-B (2008) Place cells, grid cells, and the brain's spatial representation system. Annu Rev Neurosci 31:69-89. CrossRef Medline

Muller RU, Kubie JL, Ranck JB Jr (1987) Spatial firing patterns of hippocampal complex-spike cells in a fixed environment. J Neurosci 7:19351950. Medline

Muller RU, Bostock E, Taube JS, Kubie JL (1994) On the directional firing properties of hippocampal place cells. J Neurosci 14:7235-7251. Medline

O'Keefe J (1976) Place units in the hippocampus of the freely moving rat. Exp Neurol 51:78-109. CrossRef Medline

O’Keefe J, Dostrovsky J (1971) The hippocampus as a spatial map. Preliminary evidence from unit activity in the freely-moving rat. Brain Res 34: 171-175. CrossRef Medline

O’Keefe J, Nadel L (1978) The hippocampus as a cognitive map. Oxford, UK: Oxford UP.

Sargolini F, Fyhn M, Hafting T, McNaughton BL, Witter MP, Moser M-B, Moser EI (2006) Conjunctive representation of position, direction, and velocity in entorhinal cortex. Science 312:758-762. CrossRef Medline

Skaggs WE, McNaughton BL, Gothard KM, Markus EJ (1993) An information-theoretic approach to deciphering the hippocampal code. In: Advances in neural information processing systems 5 (Hanson SJ, Cowan JD, Giles CL), pp 1030-1037. San Mateo, CA: Morgan Kaufmann.

Taube JS (2007) The head direction signal: origins and sensory-motor integration. Annu Rev Neurosci 30:181-207. CrossRef Medline

Taube JS, Muller RU, Ranck JB Jr (1990) Head-direction cells recorded from the postsubiculum in freely moving rats. I. Description and quantitative analysis. J Neurosci 10:420-435. Medline

Ulanovsky N, Moss CF (2007) Hippocampal cellular and network activity in freely moving echolocating bats. Nat Neurosci 10:224-233. CrossRef Medline

Ulanovsky N, Moss CF (2011) Dynamics of hippocampal spatial representation in echolocating bats. Hippocampus 21:150-161. CrossRef Medline

Wiener SI, Taube JS (2005) Head direction cells and the neural mechanisms of spatial orientation. Cambridge, MA: Bradford Books.

Wiener SI, Paul CA, Eichenbaum H (1989) Spatial and behavioral correlates of hippocampal neuronal activity. J Neurosci 9:2737-2763. Medline

Wills TJ, Cacucci F, Burgess N, O'Keefe J (2010) Development of the hippocampal cognitive map in preweanling rats. Science 328:1573-1576. CrossRef Medline

Wilson MA, McNaughton BL (1993) Dynamics of the hippocampal ensemble code for space. Science 261:1055-1058. CrossRef Medline

Wood ER, Dudchenko PA, Robitsek RJ, Eichenbaum H (2000) Hippocampal neurons encode information about different types of memory episodes occurring in the same location. Neuron 27:623-633. CrossRef Medline

Yartsev MM, Witter MP, Ulanovsky N (2011) Grid cells without theta oscillations in the entorhinal cortex of bats. Nature 479:103-107. CrossRef Medline

Zar JH (2010) Biostatistical analysis. Upper Saddle River, NJ: Prentice Hall. 